\title{
The VIMOS Public Extragalactic Redshift Survey (VIPERS) ${ }^{\star}$
}

\section{An unprecedented view of galaxies and large-scale structure at $0.5<z<1.2$}

\author{
L. Guzzo ${ }^{1,2}$, M. Scodeggio ${ }^{3}$, B. Garilli ${ }^{3,4}$, B. R. Granett ${ }^{1}$, A. Fritz ${ }^{3}$, U. Abbas ${ }^{5}$, C. Adami ${ }^{4}$, S. Arnouts ${ }^{4,6}$, J. Bel $^{7,1}$, \\ M. Bolzonella ${ }^{8}$, D. Bottini ${ }^{3}$, E. Branchini ${ }^{9,26,27}$, A. Cappi ${ }^{8,28}$, J. Coupon ${ }^{11,29}$, O. Cucciati $^{8,16}$, I. Davidzon ${ }^{8,16}$, \\ G. De Lucia ${ }^{12}$, S. de la Torre ${ }^{13}$, P. Franzetti ${ }^{3}$, M. Fumana ${ }^{3}$, P. Hudelot ${ }^{18}$, O. Ilbert ${ }^{4}$, A. Iovino ${ }^{1}$, J. Krywult ${ }^{14}$, \\ V. Le Brun ${ }^{4}$, O. Le Fèvre ${ }^{4}$, D. Maccagni ${ }^{3}$, K. Małek ${ }^{15}$, F. Marulli ${ }^{16,17,8}$, H. J. McCracken ${ }^{18}$, L. Paioro ${ }^{3}$, J. A. Peacock ${ }^{13}$, \\ M. Polletta ${ }^{3}$, A. Pollo ${ }^{20,21}$, H. Schlagenhaufer ${ }^{22,19}$, L. A. M. Tasca ${ }^{4}$, R. Tojeiro ${ }^{10}$, D. Vergani ${ }^{23}$, G. Zamorani $^{8}$, \\ A. Zanichelli ${ }^{24}$, A. Burden ${ }^{10}$, C. Di Porto ${ }^{8}$, A. Marchetti ${ }^{1,25}$, C. Marinoni ${ }^{7}$, Y. Mellier ${ }^{18}$, L. Moscardini ${ }^{16,17,8}$, \\ R. C. Nichol ${ }^{10}$, W. J. Percival ${ }^{10}$, S. Phleps ${ }^{19}$, and M. Wolk ${ }^{18}$
}

(Affiliations can be found after the references)

Received 16 March 2013 / Accepted 10 March 2014

\begin{abstract}
We describe the construction and general features of VIPERS, the VIMOS Public Extragalactic Redshift Survey. This ESO Large Programme is using the Very Large Telescope with the aim of building a spectroscopic sample of $\sim 100000$ galaxies with $i_{A B}<22.5$ and $0.5<z<1.5$. The survey covers a total area of $\sim 24 \mathrm{deg}^{2}$ within the CFHTLS-Wide W1 and W4 fields. VIPERS is designed to address a broad range of problems in large-scale structure and galaxy evolution, thanks to a unique combination of volume $\left(\sim 5 \times 10^{7} h^{-3} \mathrm{Mpc}^{3}\right)$ and sampling rate ( 40\%), comparable to state-of-the-art surveys of the local Universe, together with extensive multi-band optical and near-infrared photometry. Here we present the survey design, the selection of the source catalogue and the development of the spectroscopic observations. We discuss in detail the overall selection function that results from the combination of the different constituents of the project. This includes the masks arising from the parent photometric sample and the spectroscopic instrumental footprint, together with the weights needed to account for the sampling and the success rates of the observations. Using the catalogue of 53608 galaxy redshifts composing the forthcoming VIPERS Public Data Release 1 (PDR-1), we provide a first assessment of the quality of the spectroscopic data. The stellar contamination is found to be only $3.2 \%$, endorsing the quality of the star-galaxy separation process and fully confirming the original estimates based on the VVDS data, which also indicate a galaxy incompleteness from this process of only $1.4 \%$. Using a set of 1215 repeated observations, we estimate an rms redshift error $\sigma_{z} /(1+z)=4.7 \times 10^{-4}$ and calibrate the internal spectral quality grading. Benefiting from the combination of size and detailed sampling of this dataset, we conclude by presenting a map showing in unprecedented detail the large-scale distribution of galaxies between 5 and 8 billion years ago.
\end{abstract}

Key words. cosmology: observations - large-scale structure of Universe - galaxies: distances and redshifts - galaxies: statistics

\section{Introduction}

One of the major achievements of observational cosmology in the 20th century has been the detailed reconstruction of the large-scale structure of what is now called the "local Universe" $(z \leq 0.2)$. Large redshift surveys such as the 2dFGRS (Colless et al. 2001) and SDSS (York et al. 2000; Abazajian et al. 2009) have assembled samples of over a million objects, precisely characterising large-scale structure in the nearby Universe on scales ranging from 0.1 to $100 h^{-1} \mathrm{Mpc}$. The SDSS in particular is still extending its reach, using luminous red galaxies (LRG) as

* Based on observations collected at the European Southern Observatory, Cerro Paranal, Chile, using the Very Large Telescope under programmes 182.A-0886 and partly 070.A-9007. Also based on observations obtained with MegaPrime/MegaCam, a joint project of CFHT and CEA/DAPNIA, at the Canada-France-Hawaii Telescope (CFHT), which is operated by the National Research Council (NRC) of Canada, the Institut National des Sciences de l'Univers of the Centre National de la Recherche Scientifique (CNRS) of France, and the University of Hawaii. This work is based in part on data products produced at TERAPIX and the Canadian Astronomy Data Centre as part of the Canada-France-Hawaii Telescope Legacy Survey, a collaborative project of NRC and CNRS. The VIPERS website is http: //www.vipers.inaf.it/ highly effective dilute tracers of large volumes (Eisenstein et al. 2011; Ahn et al. 2012).

In addition to changing our view of the galaxy distribution around us, the quantitative analysis of galaxy redshift surveys has consistently yielded important advances in our knowledge of the cosmological model. Galaxy clustering on large scales is one of the most important relics of the initial conditions that shaped our Universe, and the observed shape of the power spectrum $P(k)$ of density fluctuations (or of its Fourier transform, the correlation function $\xi(r))$ indicates that we live in a low-density Universe in which only $25-30 \%$ of the mass-energy density is provided by (mostly dark) matter. Combined with other observations, particularly anisotropies in the cosmic microwave background (CMB), this observation has long argued for the rejection of open models in favour of a flat universe dominated by a negative-pressure cosmological constant (Efstathiou et al. 1990). This conclusion predated the more direct demonstration via the Hubble diagram of distant Type Ia Supernovae (Riess et al. 1998; Perlmutter et al. 1999) that the Universe is currently in a phase of accelerated expansion. Subsequent large-scale structure and CMB data (e.g. Cole et al. 2005; Komatsu et al. 2009; Hinshaw et al. 2013) have only reinforced the conclusion that the Universe is dominated by a repulsive "dark energy". Current observations 
are consistent with the latter being in the simplest form already suggested by Einstein with his Cosmological Constant, i.e. a fluid with non-evolving equation of state $w=-1$.

Theoretical difficulties with the cosmological constant, specifically the smallness and fine-tuning problems (e.g. Weinberg 1989) make scenarios with evolving dark energy an appealing alternative. This is the motivation for projects aiming at detecting a possible evolution of $w(z)$. Redshift surveys are playing a crucial role in this endeavour, in particular after the discovery of the signature of baryonic acoustic oscillations (BAO) from the pre-recombination plasma into large-scale structure. This "standard rod" on a comoving scale of $\sim 150 \mathrm{Mpc}$ (Percival et al. 2001; Cole et al. 2005; Eisenstein et al. 2005) provides us with a powerful mean to measure the expansion history $H(z)$ via the angular diameter distance (e.g. Percival et al. 2010; Blake et al. 2011a; Anderson et al. 2012).

An even more radical explanation of the observed accelerated expansion could be a breakdown of General Relativity (GR) on cosmological scales (see e.g. Carroll et al. 2004; Jain $\&$ Khoury 2010). Such a scenario is fully degenerate with dark energy in terms of $H(z)$, a degeneracy that in principle can be lifted by measuring the growth rate of structure, which depends on the specific theory describing gravity.

There are in principle several experimental ways to measure the growth of structure. Galaxy peculiar motions, in particular, directly reflect such growth. When the redshift is used as a distance proxy, they produce a measurable effect on clustering measurements, what we call redshift-space distortions (RSD, Kaiser 1987). The anisotropy of statistical measurements like the two-point correlation function is proportional to the growth rate of cosmic structure $f(z)$, which is a trademark of the gravity theory: if GR holds, we expect to measure a growth rate $f(z)=\left[\Omega_{\mathrm{M}}(z)\right]^{0.55}$ (Peebles 1980; Lahav et al. 1991). If gravity is modified on large scales, different forms are predicted (e.g. Dvali et al. 2000; Linder \& Cahn 2007). In fact, although the RSD effect has been well known since the late 1980s (Kaiser 1987), its potential in the context of dark energy and modified gravity has become clear only recently (Guzzo et al. 2008; Zhang et al. 2007). The RSD method is now considered to be one of the most promising probes for future dark energy experiments, as testified by the exponential growth in the number of works on both measurements (e.g. Beutler et al. 2012; Blake et al. 2011a; Reid et al. 2012), and theoretical modelling (e.g. Song \& Percival 2009; Percival \& White 2009; White et al. 2009; Scoccimarro 2004; Taruya et al. 2010; Kwan et al. 2012; Reid \& White 2011; de la Torre \& Guzzo 2012). Redshift surveys are thus expected to be as important for cosmology in the present Century as they were in the previous one, as suggested by their central role in several planned experiments - especially the ESA dark-energy mission, Euclid (Laureijs et al. 2011).

The scientific yield of a redshift survey, however, extends well beyond fundamental cosmological aspects. It is equally important to achieve an understanding of the relationship between the observed baryonic components in galaxies and the darkmatter haloes that host them. For this purpose, we need to build statistically complete samples of galaxies with measured positions, luminosity, spectral properties and (typically) colours and stellar masses; in providing such data, redshift surveys are thus a vital probe of galaxy formation and evolution. Significant statistical progress has been made in relating the galaxy distribution to the underlying dark matter, via "halo occupation distribution" (HOD) modelling (Seljak 2000; Peacock \& Smith 2000; Cooray \& Sheth 2002), of accurate estimates of the galaxy twopoint correlation function, for samples selected in luminosity, colour and stellar mass (e.g. Zehavi et al. 2004). At the same time, important global galaxy population trends involving properties such as luminosities, stellar masses, colours and structural parameters can be precisely measured when these parameters are available for $\sim 10^{6}$ objects, as in the case of the SDSS (e.g. Kauffmann et al. 2003).

In more recent years, deeper redshift surveys over areas of 1-2 $\mathrm{deg}^{2}$ have focused on exploring how this detailed picture emerged from the distant past. This was the direct consequence of the development during the 1990s of multi-object spectrographs on $8-\mathrm{m}$ class telescopes. The most notable projects of this kind have been the VIMOS VLT Deep Survey (VVDS; Le Fèvre et al. 2005), the DEEP2 survey (Coil et al. 2008) and the zCOSMOS survey (Lilly et al. 2009), which adopted various strategies aimed at covering an extended redshift range, up to $z \sim 4.5$. Such depths inevitably limit the angular size and thus the volume explored in a given redshift interval, reflecting the desire of these projects to trace galaxy evolution back to its earliest phases, while understanding its relationship with environment over a limited range of scales ${ }^{1}$. Evolutionary trends in the dark-matter/galaxy connection were explored using these surveys (Zheng et al. 2007; Abbas et al. 2010), but none of these samples had sufficient volume to produce stable and reliable comparisons of e.g. the amplitude and shape of the correlation function. Only the Wide extension of VVDS (Garilli et al. 2008), started to have sufficient volume as to attempt cosmologically meaningful computations at $z \sim 1$ (Guzzo et al. 2008), albeit with large error bars. In general, clustering measurements at $z \sim 1$ from these samples remained dominated by field-tofield fluctuations (cosmic variance), as dramatically shown by the discrepancy observed between the VVDS and zCOSMOS correlation function estimates at $z \simeq 0.8$ (de la Torre et al. 2010).

At the end of the past decade it was therefore clear that a new step in deep redshift surveys was needed, if these were to produce statistical results that could be compared on an equal footing with those derived from surveys of the local Universe, such as $2 \mathrm{dFGRS}$ and SDSS. Following those efforts, new generations of cosmological surveys have focused on covering the largest possible volumes at intermediate depths, utilizing relatively lowdensity tracers, with the main goal of measuring the BAO signal at redshifts 0.4-0.8. This is the case with the SDSS-3 BOSS project (Eisenstein et al. 2011; Dawson et al. 2013), which extends the concept pioneered by the SDSS selection of LRG (e.g. Anderson et al. 2012; Reid et al. 2012). Similarly, the WiggleZ survey further exploited the long-lived $2 \mathrm{dF}$ positioner on the AAT 4-m telescope, to target emission-line galaxies selected from UV observations of the GALEX satellite (Drinkwater et al. 2010; Blake et al. 2011a,b). Both these surveys are characterised by a very large volume $\left(1-2 h^{-3} \mathrm{Gpc}^{3}\right)$, and a relatively sparse galaxy population $\left(\sim 10^{-4} h^{3} \mathrm{Mpc}^{-3}\right)$. This is typical of surveys performed with fibre positioning spectrograph, which normally can observe 500-1000 galaxies over areas of 1-2 square degrees. Higher galaxy densities can be achieved with such systems via multiple visits, although this then limits the redshift

1 The PRIMUS survey (Coil et al. 2011) is a notable recent addition, with $\sim 120000$ spectra for galaxies at $z<1$, collected over 7 fields for a total area of $9 \mathrm{deg}^{2}$. Redshifts are obtained with a low-resolution prism (Cool et al. 2013), yielding typical errors one order of magnitude larger than those of the VIMOS surveys (see also Sect. 5.3). As such, analyses of these data have concentrated on galaxy evolution studies requiring lower precision on galaxy distances. Nevertheless, while we were revising this paper, a first detailed study of the clustering of galaxies as a function of luminosity and colour was published in the arXiv (Skibba et al. 2014). 
and/or volume surveyed. This approach has been taken by the GAMA survey (Driver et al. 2011), which aims to achieve similar numbers of redshifts to the 2dFGRS ( 200000$)$, but working to $r<19.8$ and out to $z \simeq 0.5$. Indeed, the high sampling density of GAMA makes it an important intermediate step between the local surveys and the higher redshifts probed by the survey we are presenting in this paper, i.e. VIPERS.

VIPERS stands for VIMOS Public Extragalactic Redshift Survey and has been designed to measure redshifts for approximately 100000 galaxies at a median redshift $z \simeq 0.8$. The central goal of this strategy is to build a data set capable of achieving an order of magnitude improvement on the key statistical descriptions of the galaxy distribution and internal properties, at an epoch when the Universe was about half its current age. Such a data set would allow combination with local samples on a comparable statistical footing. Despite being centred at $\bar{z} \sim 0.7$, in terms of volume and number density VIPERS is similar to local surveys like 2dFGRS and SDSS. All these surveys are characterised by a high sampling density, compared to the sparser samples of the recent generation of BAO-oriented surveys.

In this paper we provide an overview of the VIPERS survey design and strategy, discussing in some detail the construction of the target sample. The layout of the paper is as follows: in Sect. 2, we discuss the survey design; in Sect. 3 we describe the properties of the VIPERS parent photometric data and the build-up of a homogeneous sample over $24 \mathrm{deg}^{2}$; in Sect. 4 we discuss how from these data the specific VIPERS target sample at $z>0.5$ has been selected, using galaxy colours; in Sect. 5 the details of the VIMOS observations and the general properties of the spectroscopic sample are presented; in Sect. 6 we discuss the various selection effects and how they have been accounted for; finally, in Sect. 7 we present the redshift and large-scale spatial distribution of the current sample, summarising the scientific investigations that are part of separate papers currently submitted or in preparation.

As a public survey, we hope and expect that the range of science that will emerge from VIPERS will greatly exceed the core analyses from the VIPERS Team. This paper is therefore also to introduce the new VIPERS data, in view of the first Public Data Release (PDR-1) ${ }^{2}$, which is described in more detail in the specific accompanying paper (Garilli et al. 2014).

\section{Survey design}

VIPERS was conceived in 2007 with a focus on clustering and RSD at $z \simeq 0.5-1$, but with a desire to enable broader goals involving large-scale structure and galaxy evolution, similarly to the achievements of $2 \mathrm{dFGRS}$ and SDSS at $z \simeq 0.1$. The survey design was also strongly driven by the specific features of the VIMOS spectrograph, which has a relatively small field of view compared to fibre positioners $\left(\simeq 18 \times 16 \mathrm{arcmin}^{2}\right.$; see Sect. 6), but a larger yield in terms of redshifts per unit area.

Given the luminosity function of galaxies and results from previous VIMOS surveys as VVDS (Le Fèvre et al. 2005; Garilli et al. 2008) and zCOSMOS (Lilly et al. 2009), we knew that a magnitude-limited sample with $i_{A B}<22.5-23.0$ would cover the redshift range out to $z \sim 1.2$, and could be assembled with fairly short VIMOS exposure times $(<1 \mathrm{~h})$. Also, taking $2 \mathrm{dFGRS}$ as a local reference, a comparable survey volume $\sim 5 \times 10^{7} h^{-3} \mathrm{Mpc}^{3}$ could have been covered by mapping at this depth an area of $\sim 25 \mathrm{deg}^{2}$. The first attempt towards this kind of survey was VVDS-Wide, which covered $\sim 8 \mathrm{deg}^{2}$ down to a magnitude

\footnotetext{
2 Available at http://vipers.inaf.it
}

$i_{A B}=22.5$, but observing all kinds of objects (stars and galaxies), with low sampling ( $\simeq 20 \%)$.

Building upon this experience, VIPERS was designed to maximise the number of galaxies observed in the range of interest, i.e. at $z>0.5$, while at the same time attempting to select against stars, which represented a contamination up to $30 \%$ in some of the VVDS-Wide fields. The latter criterion requires multi-band photometric information and excellent seeing quality, but these qualities also benefit the galaxy sample, where a wider range of ancillary science is enabled if the galaxy surface-brightness profiles can be well resolved. The outstanding imaging dataset that was available for these purposes was the Canada-France-Hawaii Telescope Legacy Survey (CFHTLS) Wide photometric catalogue, as described below in Sect. 3.

The desired redshift range was isolated through a simple and robust colour-colour selection on the $(r-i)$ vs. $(u-g)$ plane (as shown in Fig. 3). This is one of many ways in which we have been able to benefit from the experience of previous VIMOS spectroscopic surveys: we could be confident in advance that this selection method would efficiently remove galaxies at $z<0.5$, while yielding $>98 \%$ completeness for $z>0.6$, as verified in the results shown below. A precise calibration of this separation method was made possible by the location of the VVDSWide $\left(i_{A B}<22.5\right)$ and VVDS-Deep $\left(i_{A B}<24\right)$ samples within the W4 and W1 fields of CFHTLS, respectively. This was an important reason for locating the VIPERS survey areas within these two CFHTLS fields while partly overlapping the original VVDS areas, as shown in Fig. 1. The magnitude limit was set as in VVDS-Wide, i.e. $17.5 \leq i_{A B} \leq 22.5$ (after correction for Galactic extinction).

The details of the star-galaxy separation are discussed in Appendix A, while the colour-colour selection is described in Sect. 4.

\section{Photometric source catalogue}

The VIPERS target selection is derived from the 'T0005' release of the CFHTLS Wide which was available for the first observing season 2007/2008. This object selection was completed and improved using the subsequent T0006 release, as we describe in the following.

The mean limiting AB magnitudes of CFHTLS Wide (corresponding to the $50 \%$ completeness for point sources) are $\sim 25.3,25.5,24.8,24.48,23.60$ in $u^{*}, g^{\prime}, r^{\prime}, i^{\prime}, z^{\prime}$, respectively. To construct the CFHTLS catalogues used here, objects in each tile were detected on a gri- $\chi^{2}$ image (Szalay et al. 1999) and galaxies were selected using SEXtractor's "mag_auto" magnitudes (Bertin \& Arnouts 1996), in the AB system ${ }^{3}$. These are the magnitudes used throughout this work, after they have been corrected for foreground Galactic extinction using the following prescription:

$$
\begin{aligned}
u & =u_{\text {raw }}^{*}-4.716 * E(B-V) \\
g & =g_{\text {raw }}^{\prime}-3.654 * E(B-V) \\
r & =r_{\text {raw }}^{\prime}-2.691 * E(B-V) \\
i & =i_{\text {raw }}^{\prime}-1.998 * E(B-V) \\
z & =z_{\text {raw }}^{\prime}-1.530 * E(B-V),
\end{aligned}
$$

where the extinction factor $E(B-V)$ is derived at each galaxy's position from the Schlegel dust maps (Schlegel et al. 1998).

3 http://terapix.iap.fr/rubrique.php?id_rubrique $=252$ 

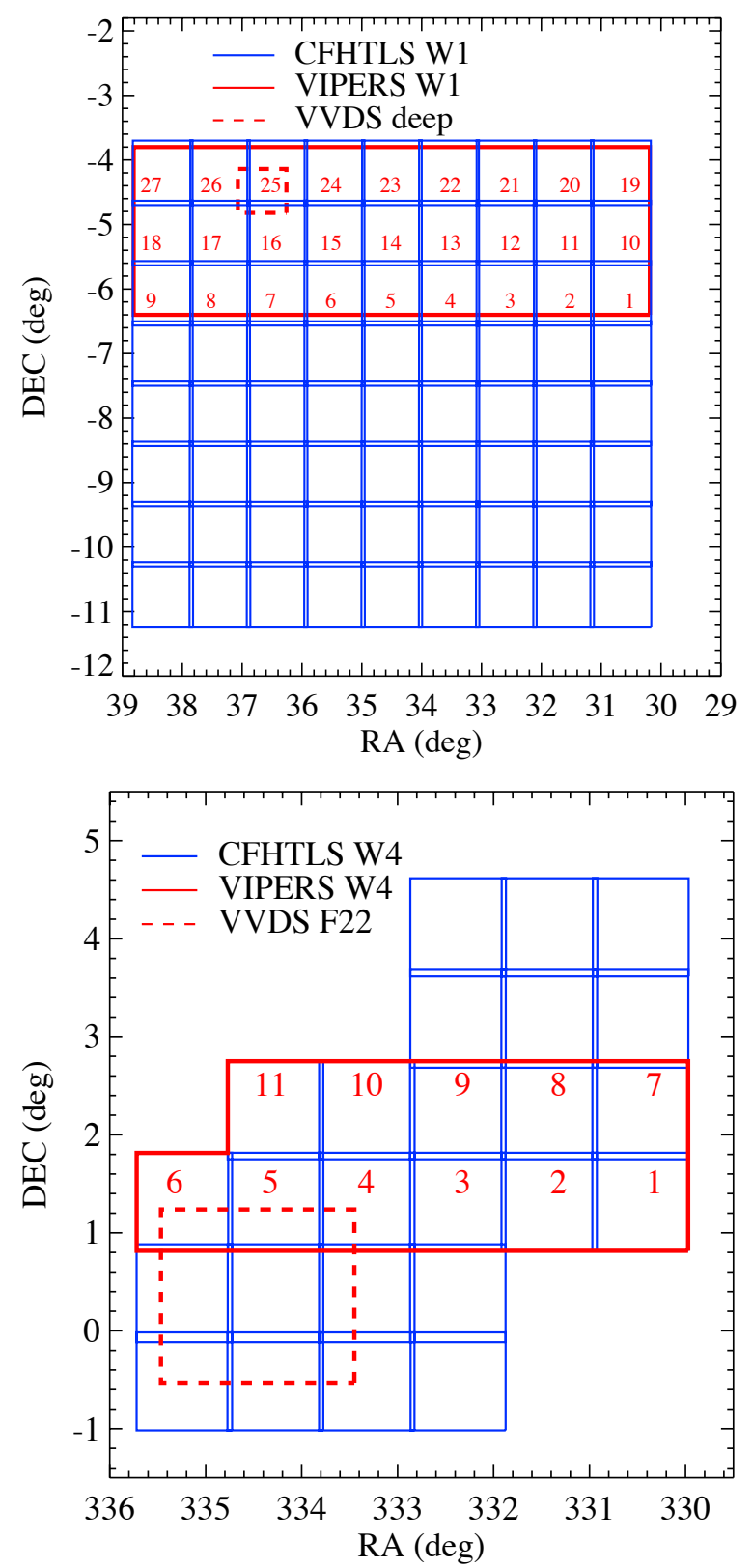

Fig. 1. Areas covered by VIPERS within the CFHTLS-Wide W1 (top) and W4 (bottom) fields. The internal numbering reported on each tile is linked to the CFHTLS naming convention in Tables C.1 and C.2. Also shown are the positions of the VVDS-Deep (Le Fèvre et al. 2005) and VVDS-Wide (Garilli et al. 2008) survey fields.

When the first target catalogues were generated, the CFHTLS survey included some photometrically incomplete areas ("holes" hereafter). In these areas one or more bands was either corrupted or missing. In particular, all of the VIPERS W1 field at right ascensions less than $\mathrm{RA} \simeq 02^{\mathrm{h}} 09^{\prime}$ were missing one band as CFHTLS Wide observations had not been completed. Smaller survey holes were mostly due to the partial failure of amplifier electronics (since all CCDs have two outputs, some images are missing only half-detector areas).

In general, these missing bands meant that we were not able to select VIPERS targets in the affected areas and they were therefore excluded from our first two observing seasons (2008 and 2009). The majority of these problems were fixed in Summer 2010 using the CFHTLS-T0006, which was carefully merged with the existing VIPERS target list. The T0005 and T0006 catalogs, limited to $i_{A B}<23.0$, were positionally matched over the area of each hole, using a search radius of 0.6 arcsec. All matches with a compatible $i$-band magnitude (defined as having a difference less than $0.2 \mathrm{mag}$ ) were considered as good identifications and used to verify the consistency between the two releases.

For objects near the VIPERS faint limit, i.e. $i_{A B} \sim 22.5$, the rms magnitude offset between the two catalogues was found to range between 0.02 to 0.04 mag (larger in the $u$-band), and smaller than this for brighter objects. Given this result, we concluded that the T0006 version of galaxy magnitudes could be used directly to replace the bad or missing magnitudes for the original T0005 objects in the holes. This solution was definitely preferable to replacing all magnitudes with their T0006 values, an operation that would have modified the target sample at the faint limit simply due to statistical scatter.

Only a few of the T0005 holes arising from CCD failures were not filled by the T0006 release. To complete these remaining areas, Director's Discretionary Time (DDT) was awarded at CFHT with MegaCam in summer 2009 (Arnouts \& Guzzo, priv. comm.). At the end of 2010, the combination of new T0006 observations and the DDT data resulted in a virtually complete coverage in all five bands of the two VIPERS areas in W1 and W4. The last problem to be resolved was re-calibrating a few small areas which were observed in T0006 with a new $i$-band filter, called " $y$ ", as the original $i$-band filter broke in 2007. This procedure is described in Appendix B.

\subsection{Tile-to-tile zero-point homogenisation}

The CFHTLS data are provided in single tiles of $\sim 1 \mathrm{deg}$ side, overlapping each other by $\sim 2$ arcmin to allow for crosscalibration. These are shown in Fig. 1 for the W1 and W4 fields, together with the position of the two VIPERS areas. To build the VIPERS global catalogue we merged adjacent tiles, eliminating duplicated objects. In these cases, the object in a pair having the best Terapix flag was chosen; if the flags were identical, the object at the greater distance from the tile border was chosen. Tiles were merged proceeding first in right ascension rows and then merging the rows into a single catalogue.

For any galaxy survey planning to measure large-scale clustering it is crucial that the photometric or colour selection is as homogeneous as possible over the full survey area in order to avoid creating spurious object density fluctuations that could be mistaken as real inhomogeneities. Given the way the CFHTLSWide catalogue has been assembled, verifying and correcting any tile-to-tile variation of this kind is therefore of utmost importance. In fact, it was known and directly verified that each tile in T0005 still had a small but non-negligible zero-point offset in some of the photometric bands. These offsets are a consequence of non-photometric images being used as photometric anchor fields in the global photometric solution.

These tile-to-tile colour variations are evident when stars are plotted in a colour-colour diagram, as in Fig. 2. In this figure we show the $(u-g)$ vs. $(r-i)$ colours for stellar objects in two particularly discrepant tiles (see Appendix A for details on how stars and galaxies are separated). Such offsets can produce two kinds of systematic effects in a survey like VIPERS. First, a tile-to-tile difference in the selection magnitude ( $i$ band) would introduce a varying survey depth over the sky and thus a variation in the expected number counts and redshift distribution. Secondly, the colours would be affected, and thus any colour-colour selection (as the one applied to select galaxies at $z>0.5$ for the VIPERS 


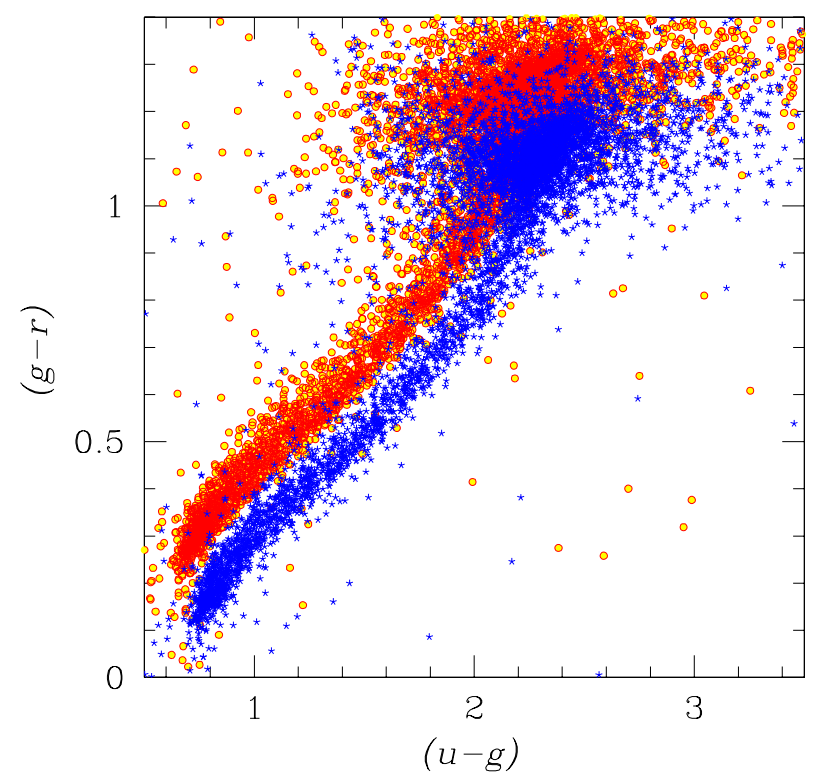

Fig. 2. One of largest tile-to-tile magnitude zero-point variations in the T0005 data. The position of the stellar sequence in the $(g-r)$ vs. $(u-g)$ plane is compared for tile \#9 and tile \#11 in the W4 VIPERS area (see Appendix C), showing an offset of $\sim 0.15 \mathrm{mag}$ in $(g-r)$ and $\sim 0.06$ in $(u-g)$ between the two tiles.

target catalogue - see next section), would vary from one tile to another.

The well-defined location of stars in colour-colour space, as shown in Fig. 2, suggests a technique for a possible correction of the colour variations, i.e. using the observed stellar sequence as a colour calibrator (see High et al. 2009, for a similar more recent application of this regression technique). An important assumption of this correction procedure is that stars and galaxies are affected by similar zero-point shifts, and thus that stellar sequences can also be used to improve the photometric calibration of extended objects. This assumption is quite reasonable and it is the same adopted at Terapix in the past to check internal calibration until the second-last release, i.e. T0006. With the latest release, T0007, there are indications that a contribution to these zero-point discrepancies could be also due to a dependence on seeing of mag_auto when applied to stellar objects. This effect is not fully understood yet and its amplitude is smaller than the corrections we originally applied to the T0005 data. The potential systematic impact of this uncertainty, in particular on clustering analyses of the PDR-1 sample, is explicitly addressed in the corresponding papers (see e.g. de la Torre et al. 2013).

The colour corrections were carried out assuming (a) that the $i$-band magnitude had a negligible variation from tile to tile, and (b) taking the colours measured in tile W1-25 (see Fig. 1) as the reference ones. W1-25 is the tile overlapping the VVDSDeep survey, which was used to calibrate the colour selection criteria as discussed in Sect. 4. By referring all colours to that tile, we assured (at least) that the colour-redshift correlation we calibrated was applied self-consistently to all tiles. For all tiles covered by VIPERS we measured therefore the $(u-g)$ value of the blue-end cut-off in the stellar sequence, clearly visible in Fig. 2, together with the zero points derived from a linear regression to the $(g-r)$ vs. $(u-g)$ and $(r-i)$ vs. $(u-g)$ relationships for stars. These two regressions give a consistent slope of 0.50 and 0.23 , respectively, over all tiles. This allowed us to compute three colour offsets $\delta_{\mathrm{ug}}, \delta_{\mathrm{gr}}$ and $\delta_{\text {ri }}$ for each tile, corresponding to the values required to match the same measurements in W1-25.

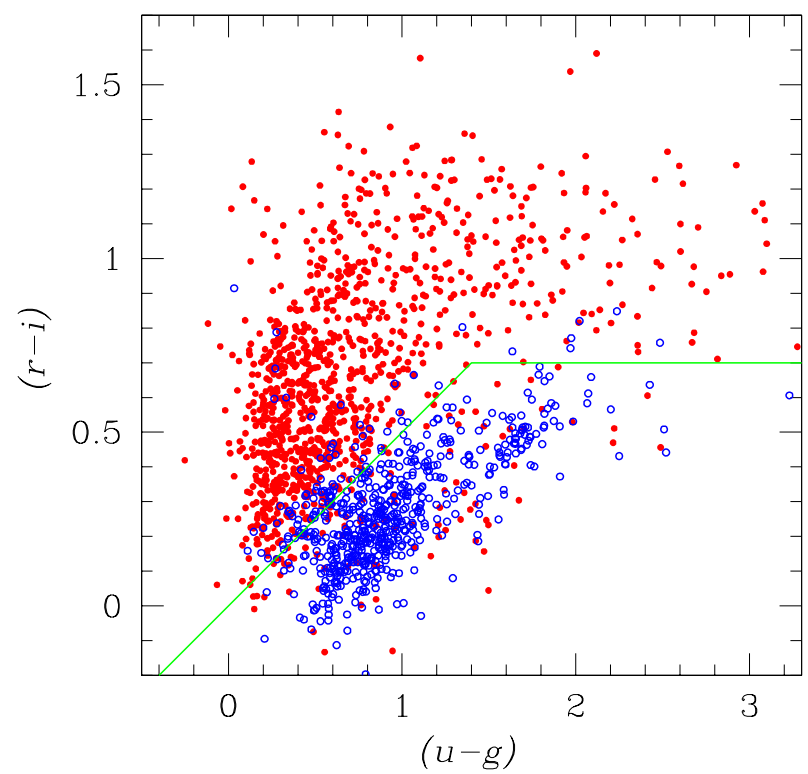

Fig. 3. Distribution in the $(r-i)$ vs. $(u-g)$ plane of $i_{A B}<22.5$ galaxies with known redshift from the VVDS-Deep survey, showing the kind of selection applied to construct the VIPERS target sample. The colour selection of Eq. (9) is described by the continuous line, which empirically splits the sample into $z>0.5$ (red filled circles) and $z<0.5$ (blue open circles) by optimising the completeness and contamination of the high-redshift sample.

All following steps in the selection of VIPERS target galaxies were then operated on colours corrected using these offsets, i.e.

$(u-g)=(u-g)_{\text {uncorr }}-\delta_{\text {ug }}$

$(g-r)=(g-r)_{\text {uncorr }}-\delta_{\text {gr }}$

$(r-i)=(r-i)_{\mathrm{uncorr}}-\delta_{\mathrm{ri}}$.

\section{Selection of VIPERS galaxy targets}

Around half of the galaxies in a magnitude-limited sample with $i_{A B}<22.5$ are at $z<0.5$. At the same time, the average number of slits that can be accommodated within one of the four VIMOS quadrants (see below) is approximately fixed, for a parent sample with a given depth and clustering. This means that in a pure magnitude limited survey with $i_{A B}<22.5$, around half of the slits would fall on $z<0.5$ galaxies. Given the original goal of VIPERS to build a sample complementary to local surveys, a strategy was devised as to select a priori only galaxies at higher redshifts, doubling in this way the sampling over the high-redshift range. Using available magnitude-limited VVDS data, a simple yet effective and robust colour selection criterion was devised through a series of experiments. The most effective criteria are shown in Fig. 3 applied to the VVDS data. Galaxies are retained in the source list if their colours obey the following relationship:

$(r-i)>0.5(u-g) \quad$ OR $\quad(r-i)>0.7$.

The resulting distribution of the true redshifts for the selected samples is shown in Fig. 4, with the corresponding level of completeness shown in Fig. 5. To compute the latter quantity, we used the VVDS data (both Deep and Wide), and plot the ratio of the numbers of objects in a VIPERS-like selected sample, to the original total redshift sample. We call this quantity the Colour Sampling Rate (CSR). As indicated by the combination of these 


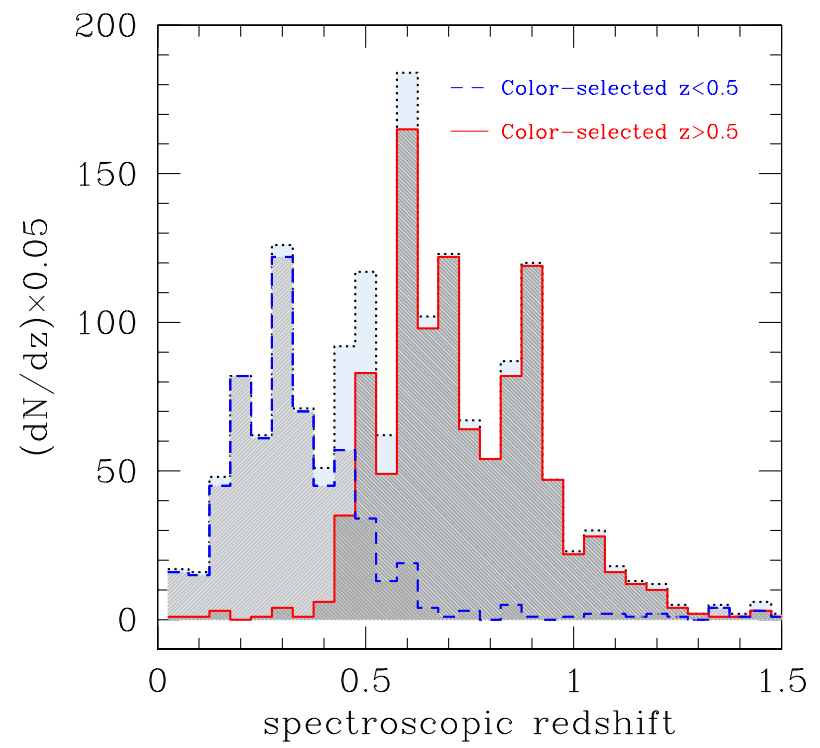

Fig. 4. Test of the colour-colour redshift selection, using galaxies with known redshift from the VVDS-Deep survey, limited to $i_{A B}<22.5$. The colour locus in Fig. 3 is used to separate a priori galaxies lying below (blue-dashed line) and above (solid red line) $z \simeq 0.5$. The dotted black line shows the global $\mathrm{d} N / \mathrm{d} z$ of the sample. The VVDS-Deep sample has been limited to objects belonging to tile \# 25 (where the bulk of the sample is concentrated), given that this has been used as the reference for the global colour calibration discussed in the text.

two figures, the VIPERS selection does not introduce any significant colour bias (i.e. it selects virtually all galaxies) above $z \sim 0.6$, with an acceptable contamination $(\sim 5 \%)$ of low-redshift interlopers. More quantitatively, some insight on the potential incompleteness of the selection procedure - i.e. on how many galaxies that should have been included in our sample at $z>0.5$ are lost - can be derived by looking in detail at the few outliers in the blue histogram of Fig. 4. The tail of unselected objects at $z>0.5$ in the VVDS calibration sample includes 46 cases. 14 of these are classified as active galactic nuclei (AGN) by the VVDS, which explains why their colour-redshift relation does not match the standard criteria defined for galaxies; 10 out of the 14 AGNs have $z>1.2$ and are thus out of the typical range used by VIPERS for statistical studies; thus only the remaining 4 could potentially be part of the VIPERS target sample, although one cannot distinguish how much the active nucleus contributes to the overall magnitude (and thus, understand whether the object would be brighter than $I_{A B}=22.5$ based on the sole magnitude of the host galaxy). 7 of the remaing outliers have an error on the $u$-band magnitude which is larger than $0.1 \mathrm{mag}$, which makes their $u-g$ colour unreliable; another 3 have a redshift flag $=1$, i.e. their redshift has a $\sim 50 \%$ probability to be wrong (see Sect. 5.3). We are left with 22 further outliers, among which 8 objects are at $z>1.2$, while (after visual inspection) another 4 show noisy spectra suggesting an incorrect redshift, thus resulting in 10 further galaxies that apparently should have been included in the target sample.

Based on these figures, a conservative upper limit to the number of galaxies missed in the range $0.5<z<1.2$ for the VIPERS-like test sample can be obtained by summing up these numbers: 4 (AGN host galaxies), plus 3 (assuming conservatively that all Flag $=1$ redshifts are correct), 7 (wrong colour), plus 10 (remaining galaxies with confirmed $z>0.5$ redshifts). This corresponds to an estimated global incompleteness of the $z>0.5$ colour/redshift selection of $24 / 1068$, i.e. $2.2 \%$. It should

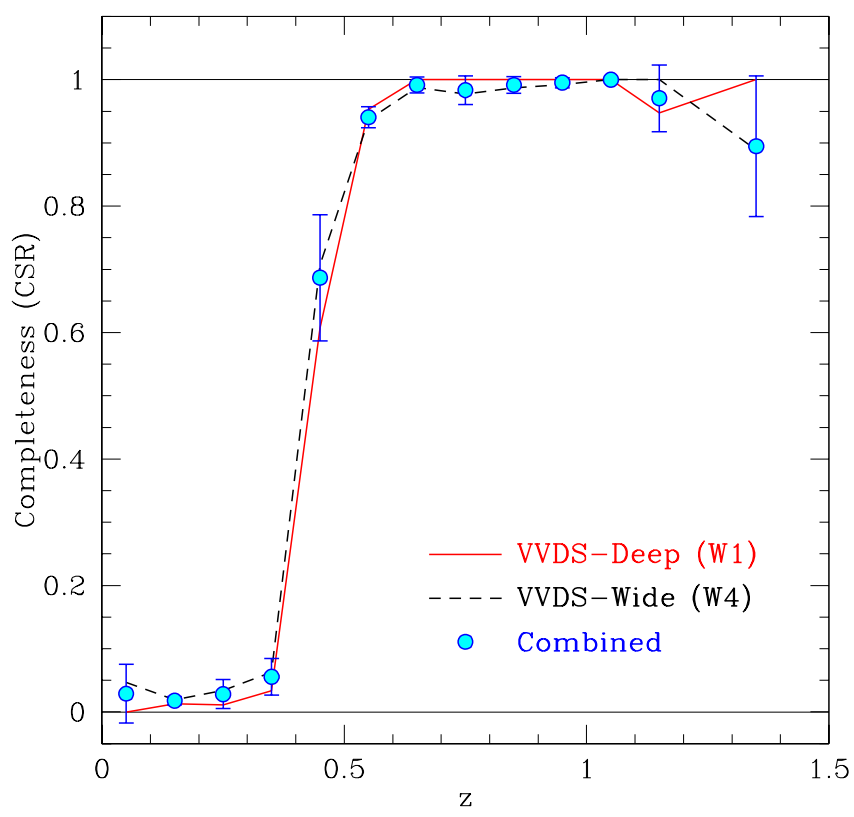

Fig. 5. Direct verification of the completeness of the VIPERS colour selection as a function of redshift, using both VVDS-Deep and VVDSWide data, in W1 and W4 respectively. Note that the original colour criteria were defined based only on the VVDS-Deep data. The curves and points give the Colour Sampling Rate (CSR), i.e. the ratio of the number of galaxies satisfying the VIPERS criteria within a redshift bin and the total number of galaxies in that same bin. Both fields provide consistent selection functions, indicating that the colour-colour selection function is basically unity above $z=0.6$ and can be consistently modelled in the transition region $0.4<z<0.6$.

be noted also that, as visible in Fig. 4, a significant fraction of this incompleteness is concentrated in the transition region $0.5<z<0.6$ and can be modelled as shown in Fig. 5 and discussed in some detail in Garilli et al. (2014).

An alternative technique to select a high-redshift sample could have been to use photometric redshifts computed using all five CFHTLS bands. We verified that this method provides comparable performance in terms of completeness and contamination to the colour-colour selection. However we preferred a simple colour-colour criterion, as it can be reproduced precisely at any time, while photometric redshifts depend inevitably also on the features of the specific codes and template selection used, which will evolve with time.

Finally, to further broaden the scientific yield of VIPERS, the galaxy target catalogue was supplemented with two small additional samples of AGN candidates. These include a sample of X-ray selected AGNs from the XMM-LSS survey in the W1 field (Pierre et al. 2007), and a sample of colour-defined AGN candidates selected among objects classified as stars in the previous phase. These two catalogues contributed on average 1-3 objects per quadrant (against about 90 galaxy targets) with negligible impact on the galaxy selection function. These AGN candidates are excluded from the current PDR-1 sample. All the details on the selection criteria and the properties of the resulting objects will be discussed in a future paper.

\section{VIMOS observations}

\subsection{The VIMOS spectrograph}

The VIPERS project is designed around the ESO VIsible MultiObject Spectrograph (VIMOS), on "Melipal", the ESO Very 

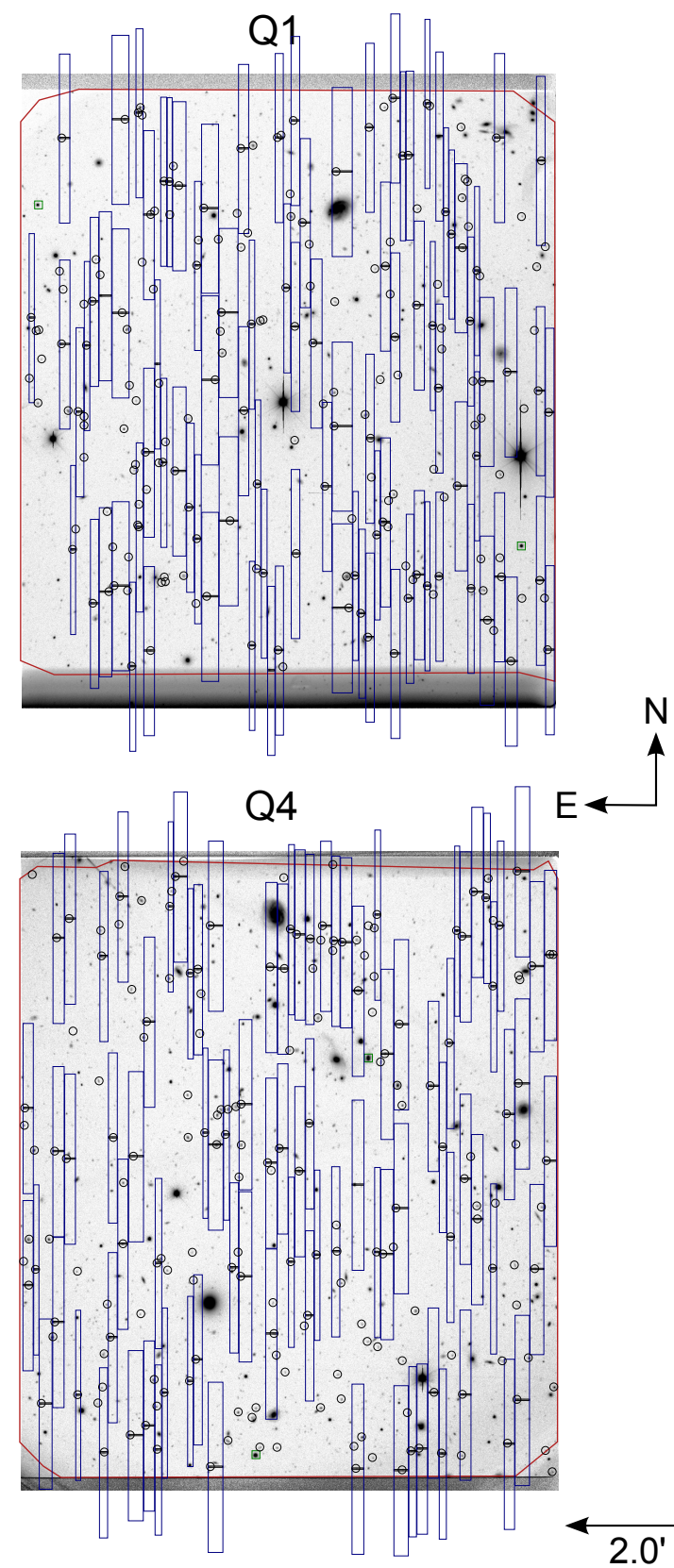

$\mathrm{N}$
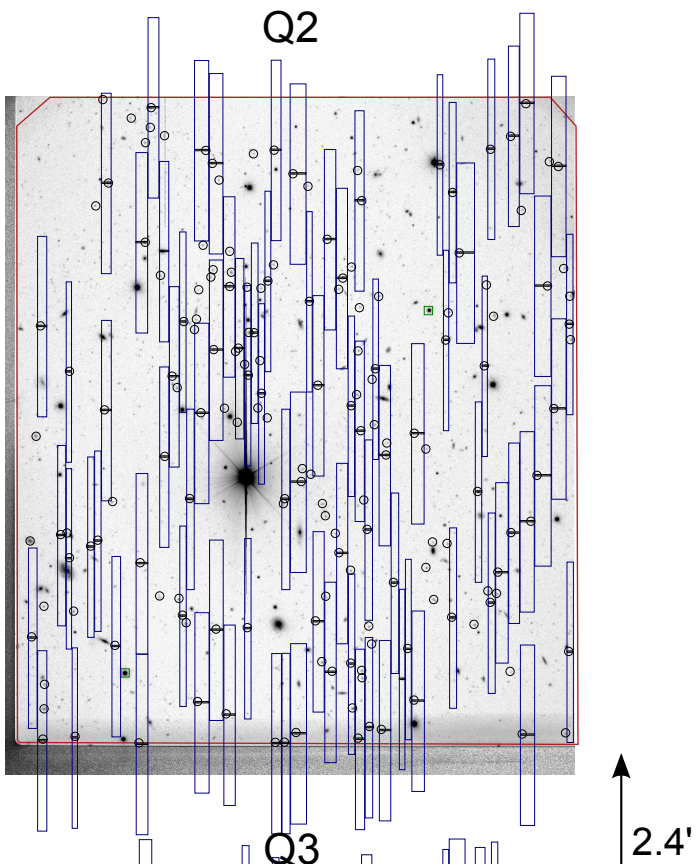

Fig. 6. Example of the detailed footprint and disposition of the four quadrants in a full VIMOS pointing (W1P082 in this case). Note the reconstructed boundaries (solid red lines), which have been traced pointing-by-pointing through an automatic detection algorithm that follows the borders of the illuminated area. These can vary in general among different pointings in the database, in particular due to the CCD refurbishment of 2010 and sometimes to vignetting by the telescope guide probe arm.

Large Telescope (VLT) Unit 3 (Le Fèvre et al. 2003). VIMOS is a 4-channel imaging spectrograph; each channel (a "quadrant") covers $\sim 7 \times 8 \operatorname{arcmin}^{2}$ for a total field of view (a "pointing") of $\sim 218 \operatorname{arcmin}^{2}$. Each channel is a complete spectrograph with the possibility to insert $\sim 30 \times 30 \mathrm{~cm}^{2}$ slit masks at the entrance focal plane, as well as broad-band filters or grisms. The standard layout of the four quadrants on the sky is reproduced in Fig. 6. The figure shows the slit positions and the resulting location of the spectra, overlaid on the direct pre-image of pointing P082 in the W1 field.

The pixel scale on the CCD detectors is 0.205 arcsec/pixel, providing excellent sampling of the Paranal mean image quality and Nyquist sampling for a slit 0.5 arcsecond in width. For the VIPERS survey, we use slits of 1 arcsecond, together with the "low-resolution red" (LR-Red) grism, which provides a spectral resolution $R \simeq 250$ over the wavelength range $\sim 5500$ $9500 \AA$. The instrument has no atmospheric dispersion compensator, given the large size of its field-of-view at the VLT Nasmyth focus $(\simeq 1 \mathrm{~m})$. For this reason, observations have to be limited to airmasses below 1.7. For VIPERS observations we rarely went above an airmass of 1.5.

To prepare the MOS masks, direct exposures ("pre-images") need to be observed beforehand under the same instrumental conditions. Object positions in these images are then crosscorrelated with the target catalogue in order match its astrometric coordinates to the actual instrument coordinate system. This operation is performed during the mask preparation using VMMPS, the standard package for automatic optimisation of the positions and total number of slits (Bottini et al. 2005). 


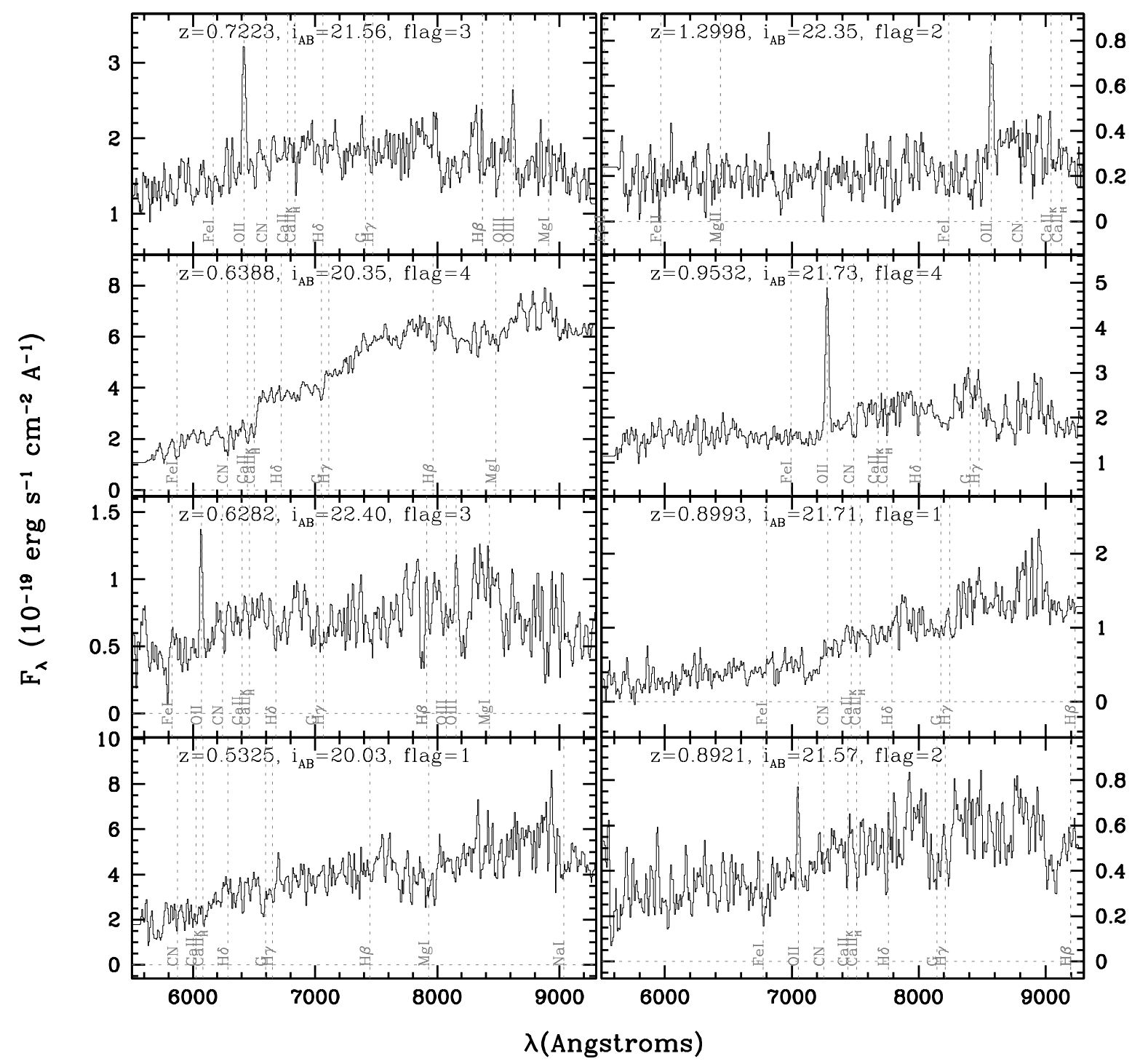

Fig. 7. A few representative examples of VIPERS spectra of early- and late-type galaxies, chosen among the different quality classes (i.e. quality flags) and at different redshifts. The quoted flux is the observed one, without corrections for finite-slit losses. The typical absorption and emission features are marked.

In summer 2010, VIMOS was upgraded with new redsensitive CCDs in each of the 4 channels, as well as with a new active flexure compensation system. The reliability of the mask exchange system was also improved (Hammersley et al. 2010). The original thinned E2V detectors were replaced by twice-thicker E2V devices, considerably lowering the fringing and increasing the global instrument efficiency by up to a factor 2.5 (one magnitude) in the redder part of the wavelength range. This upgrade significantly improved the average quality of VIPERS spectra, resulting in a significantly higher redshift measurement success rate.

\subsection{Data reduction, redshift measurement and validation}

VIPERS is the first VIMOS redshift survey for which the data reduction is performed with a fully automated pipeline, starting from the raw data and down to the calibrated spectra and redshift measurements. The pipeline includes and updates algorithms from the original VIPGI system (Scodeggio et al. 2005) within a complete purpose-built environment. Within it, the standard CCD data reduction, spectral extraction and calibration follow the usual recipes discussed in previous VIMOS papers (Le Fèvre et al. 2005; Lilly et al. 2009). The difference in the case of VIPERS is that the only operation for which we still require human intervention is the verification and validation of the measured redshift. All data reduction has been centralised in our data reduction and management centre at INAF - IASF Milano. When ready, the fully reduced data are made available to the team within a dedicated database. The full management of these operations within the "EasyLife" environment is described in Garilli et al. (2008). Figure 7 shows a few examples of VIPERS spectra, for galaxies with varying redshift and quality flag. In common with previous VIMOS surveys (e.g. Le Fèvre et al. 2005; Lilly et al. 2009), all redshifts have been validated independently by two scientists but with some simplification to increase efficiency given the very large number of spectra. Nevertheless, this required a very strong team effort. Two team members are assigned the same VIMOS field to review, with one of the two being the primary person responsible for that pointing. At the end of the process discrepant redshifts resulting from the two reviewers are discussed and reconciled. 
The quality of the measured redshifts is quantified at the time of validation through a similar grading scheme to that described in Le Fèvre et al. (2005) and Lilly et al. (2009). The corresponding confidence levels are estimated from repeated observations, as explained in Sects. 5.3 and 5.4:

- Flag 4.X: a high-confidence, highly secure redshift, based on a high signal-to-noise ratio $(\mathrm{S} / \mathrm{N})$ spectrum and supported by obvious and consistent spectral features. The combined confidence level of Flag 4 + Flag 3 measurements is estimated to be $>99 \%$

- Flag 3.X: also a very secure redshift, comparable in confidence with Flag 4, supported by clear spectral features in the spectrum, but not necessarily with high $\mathrm{S} / \mathrm{N}$.

- Flag 2.X: a fairly secure, $\sim 95 \%$ confidence redshift measurement, with sufficient spectral features in support of the measurement.

- Flag 1.X: a tentative redshift measurement, based on weak spectral features and/or continuum shape, for which there is $\sim 50 \%$ chance that the redshift is actually wrong.

- Flag 0.X: no reliable spectroscopic redshift measurement was possible.

- Flag 9.X: redshift is based on only one single clear spectral emission feature, usually identified (in the VIPERS range) with [OII] $3727 \AA$.

- Flag -10: spectrum with clear problems in the observation or data processing phases. In most cases this is a failed extraction by VIPGI (Scodeggio et al. 2005) or a bad sky subtraction because the object is too close to the edge of the slit.

Serendipitous objects appearing by chance within the slit of the main target are identified by adding a " 2 " in front of the main flag. Following human validation, a decimal fraction ".*” is added to the main flag, reflecting the agreement of the spectroscopic measurement $\left(z_{\text {spec }}\right)$, to the photometric redshift $\left(z_{\text {phot }}\right)$, estimated from the five-band CFHTLS photometry. Photometric redshifts have been derived using Le Phare (Ilbert et al. 2006; Arnouts \& Ilbert 2011), a code that provides us, on top of the best redshift solution, $z_{\text {phot }}$, with a specific $68 \%$ confidence interval $\left[\hat{z}_{\mathrm{ph}-\min }, \hat{z}_{\mathrm{ph}-\max }\right]$ for each galaxy. To quantify the level of agreement between $z_{\text {spec }}$ and $z_{\text {phot }}$ we also consider the overall error distribution that can be constructed by plotting spectroscopic and photometric redshifts against each other. Early in the survey, we adopted a value of $\sigma_{z}=0.025$ for the standard deviation (68\% interval) of the photometric redshifts, slightly smaller than the current more robust estimate using the median absolute deviation in the VIPERS data $\left(\sigma_{z}=0.03\right.$, Garilli et al. 2014). The decimal flag is defined as follows.

- We look first at whether $z_{\text {spec }}$ is included in the $95.4 \%(2 \sigma)$ interval defined by the overall statistics of photometric redshifts, i.e. the interval $z_{\text {phot }} \pm 0.05 \times\left(1+z_{\text {phot }}\right)$. In this case, there are two options:

1) if $\hat{z}_{\mathrm{ph}-\min }<z_{\mathrm{spec}}<\hat{z}_{\mathrm{ph}-\mathrm{max}}$, i.e. the spectroscopic redshift also falls within the (stricter) $68 \%$ interval of the individual PDF, this is defined as "full agreement" and a value 0.5 is added to the original (integer) flag;

2) if not, the two measurements are defined to be only in "marginal agreement", and a flag 0.4 is added.

- When neither of the previous criteria is satisfied, a value 0.2 is added.

- When no $z_{\text {phot }}$ estimate is available, a value 0.1 is added.

The rationale behind the decimal flag is to improve the confidence in poorly measured spectroscopic estimates. For example, confidence in a highly uncertain (flag $=1$ ) spectroscopic redshift, would be increased in case its comparison to $z_{\text {phot }}$ promotes it to flag $=1.5$.

In all VIPERS papers, redshifts characterised by a flag ranging between 2.X and 9.X are referred to as reliable (or secure) redshifts and are the only ones normally used in the science analyses. It might sound risky to consider objects with flag 9.2 as reliable. As explained above, these correspond to a redshift measurement based on one single emission line (normally [OII]3727 $\AA$ ), which does not agree with the galaxy photometric redshift estimate. To confim this, we inspected directly the spectra for all 1027 such cases in the PDR-1 sample. For 171 of these the single-line spectroscopic redshift is close to the photometric one, although not satisfying the statistical criteria to be defined in agreement. The vast majority ( $\sim 95 \%)$ of these cases present other features in the spectrum that allow us to promote their flag to 2 . For the remaining 856, there is no way the observed emission line could be matched to the photometric redshifts, if associated to one of the other standard galaxy emission lines.

\subsection{Error on redshift measurements}

For 783 galaxies in the VIPERS PDR-1 sample a repeated, reliable redshift measurement exists. These are objects lying at the border of the quadrants, where two quadrants overlap, and were therefore observed by two independent pointings. In addition, during the re-commissioning of VIMOS after the CCD refurbishment in summer 2010, a few pointings were re-observed to verify the performances with the new set-up (Hammersley et al. 2010), targeting another 1357 galaxies. In total, this gives a sample of 1941 galaxies with double observations. 1215 of these yield a reliable redshift (i.e. with a flag $\geq 2$ ) in both measurements and can be conveniently used to obtain an estimate of the internal rms value of the redshift error of VIPERS galaxies.

The bottom panel of Fig. 8 shows the distribution of the differences between these double measurements. The sign of these differences is clearly arbitrary. These have been computed as $z_{2}-z_{1}$, where " 1 " and " 2 " are chronologically ordered in terms of observation date. Once normalised to the corresponding redshift expansion factor $1+z$, the overall distribution of these measurements is very well described by a Gaussian with a dispersion of $\sigma_{2}=200 \mathrm{~km} \mathrm{~s}^{-1}$, corresponding to a singleobject $1 \sigma$ error $\sigma_{v}=\sigma_{2} / \sqrt{2}=141 \mathrm{~km} \mathrm{~s}^{-1}$. In terms of redshift, this yields a standard deviation on the redshift measurements of $0.00047(1+z)$. If we restrict ourselves to the highest quality spectra (i.e. flags 3 and 4), we are left with 655 double measurements; the resulting rest-frame 2-object dispersion changes very little, decreasing to $\sigma_{2}=193 \mathrm{~km} \mathrm{~s}^{-1}$. This indicates that flags 2, 3 and 4 are substantially equivalent in terms of redshift precision.

\subsection{Confidence level of quality flags}

Repeated observations allow us to quantify in an objective way the statistical meaning of our quality flags, which are by nature subjective; they are assigned by individuals in a large, geographically dispersed team. Remarkably, the grading system turns out to be quite stable and well-defined as we show hereafter.

Let us define two redshifts as "in agreement" when $\Delta z /(1+z)<3 \sigma_{z} \simeq 0.0025$. We compare the redshifts of double measurements from the VIPERS sample only, considering the flag assigned to both measurements. Flags 3 and 4 are considered together, as they should not be different in practice in terms 

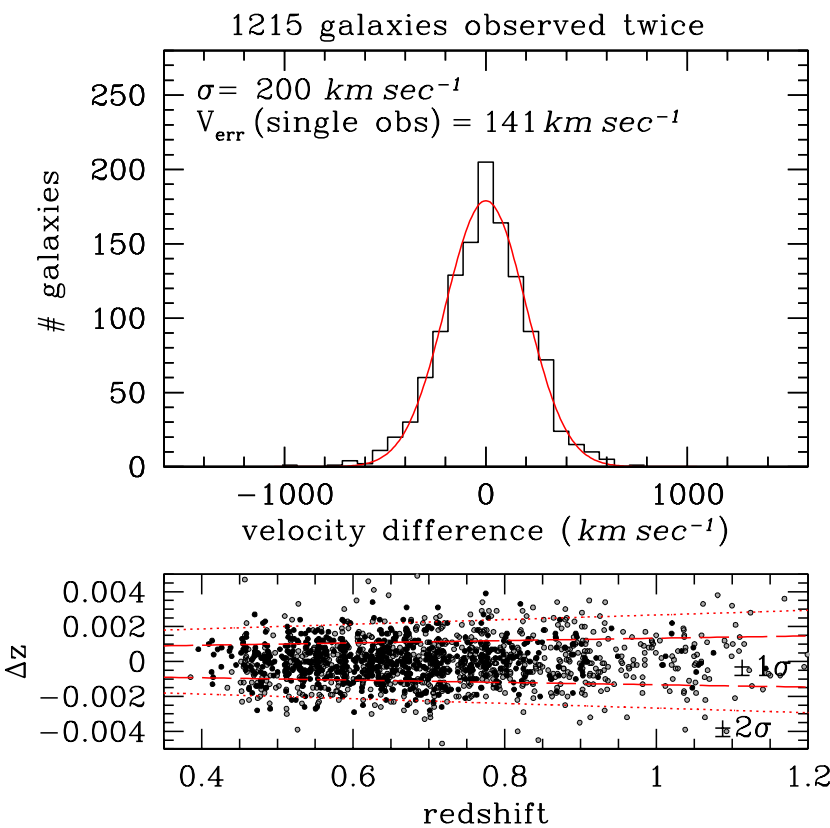

Fig. 8. Distribution of redshift differences between two independent measurements of the same object, obtained from a set of 1215 VIPERS galaxies with quality flag $\geq 2$. In the bottom panel, the darker dots correspond to top-quality redshifts (i.e. flags 3 and 4), which show a dispersion substantially similar to the complete sample (see text). Catastrophic failures (defined as being discrepant by more than $\Delta z=$ $6.6 \times 10^{-3}(1+z)$ ) have obviously been excluded. Top: distribution of the corresponding differences $\Delta v=c \Delta z /(1+z)$. The best-fitting Gaussian has a dispersion of $\sigma_{2}=200 \mathrm{~km} \mathrm{~s}^{-1}$, corresponding to a single-object rms error $\sigma_{v}=\sigma_{2} / \sqrt{2}=141 \mathrm{~km} \mathrm{~s}^{-1}$. In terms of redshift, this translates into a standard deviation of $\sigma_{z}=0.00047(1+z)$ for a single galaxy measurement.

of strict redshift reliability. We therefore consider pairs of measurements, in the following cases:

1. Both measurements have flag $=3$ or 4 : out of 655 pairs, 5 have discrepant redshift.

2. One measurement has flag $=2$ and the other $3 / 4$ : In this case we assume the measurement with flag $3 / 4$ to be the correct one. We have $10 \mathrm{flag}=2$ redshifts that are discrepant, out of 345 .

3. Both measurements have flag $=2: 22$ out of 148 pairs have discrepant redshift.

4. One measurement has flag $=1$ and the other has 2,3 or 4 : 121 out of 301 are discrepant.

5. Both measurements have flag $=1: 56$ out of 74 are discrepant.

With the reasonable assumption that when two redshifts are in agreement they are both correct, using these data we can derive a confidence level of the redshift measurements for each flag class, which we report in Table 1. A final comment should be added concerning Flag 9 objects, i.e. those redshifts based on a single emission line (tipically interpreted as [OII] $\lambda 3727$ ), in particular when they disagree with the photometric redshift (Flag 9.2). We do not have sufficient statistics for this class in the sample with repeated observations. Their reliability is discussed in more detail in the PDR-1 data release paper (Garilli et al. 2014).

\section{Survey selection function}

The VIPERS angular selection function is the result of the combination of several different angular completeness functions.
Table 1. Redshift confidence levels corresponding to the VIPERS quality flags, estimated from pairs of measurements of the same galaxy.

\begin{tabular}{lc}
\hline \hline Flag class & $z$ confidence level \\
\hline $\mathbf{3 + 4}$ & $99.6 \%$ \\
$\mathbf{2}$ & $95.1 \%$ \\
$\mathbf{1}$ & $57.5 \%$ \\
\hline
\end{tabular}

Two of these are binary masks (i.e. areas are fully used or fully lost). The first mask is related to defects in the parent photometric sample (mostly areas masked by bright stars) and the other to the specific footprint of VIMOS and how the different pointings are tailored together to mosaic the VIPERS area. Moreover, within each of the four VIMOS quadrants only an average $40 \%$ of the available targets satisfying the selection criteria are actually placed behind a slit and observed, defining what we call the Target Sampling Rate (TSR). Finally, varying observing conditions and technical issues determine a variation from quadrant to quadrant of the actual number of redshifts measured with respect to the number of targeted galaxies, what we call the Spectroscopic Success Rate (SSR).

Detailed knowledge of all these contributions is a crucial ingredient for any quantitative measurement of galaxy clustering. In principle, there will also be variations of the TSR and SSR within a single quadrant, owing to the details of the response of slit assignment to small-scale clustering, and to internal distortions that may cause the slits to be slightly misplaced on the sky. These effects are hard to represent simply, since they cannot be viewed purely as a position-dependent probability of obtaining a redshift. This is because the finite size of the slits means that close pairs of galaxies cannot be sampled, and there will always be some complex structure in the statistics of pair separations owing to the survey selection. Once the main quadrantbased corrections are made, the only practical way of dealing with these is to use the known statistics of angular clustering in the initial photometric catalogue in order to make a final small correction to the estimated clustering statistics (de la Torre et al. 2013).

\subsection{Revised CFHTLS photometric mask}

The photometric quality across the CFHTLS images is tracked with a set of masks accounting for imaging artefacts and nonuniform coverage. We use the masks to exclude regions from the survey area with corrupted source extraction or degraded photometric quality. The masks consist primarily of patches around bright stars $\left(B_{\text {Vega }}<17.5\right)$ owing to the broad diffraction pattern and internal reflections in the telescope optics. At the core of a saturated stellar halo there are no reliable detections, leaving a hole in the source catalogue, while in the halo and diffraction spikes spurious sources may appear in the catalogue due to false detections. We also add to the mask extended extragalactic sources that may be fragmented into multiple detections or that may obscure potential VIPERS sources. The masks are stored in DS9 region file format using the polygon data structure.

Terapix included a bright star mask as part of the T0006 data release consisting of star-shaped polygons centred on the stellar halos. We found this mask to be too restrictive for VIPERS; in particular, we found that the area lost was excessive near diffraction spikes and within stellar halos. We follow the same strategy in constructing the VIPERS mask, but instead use a circular 


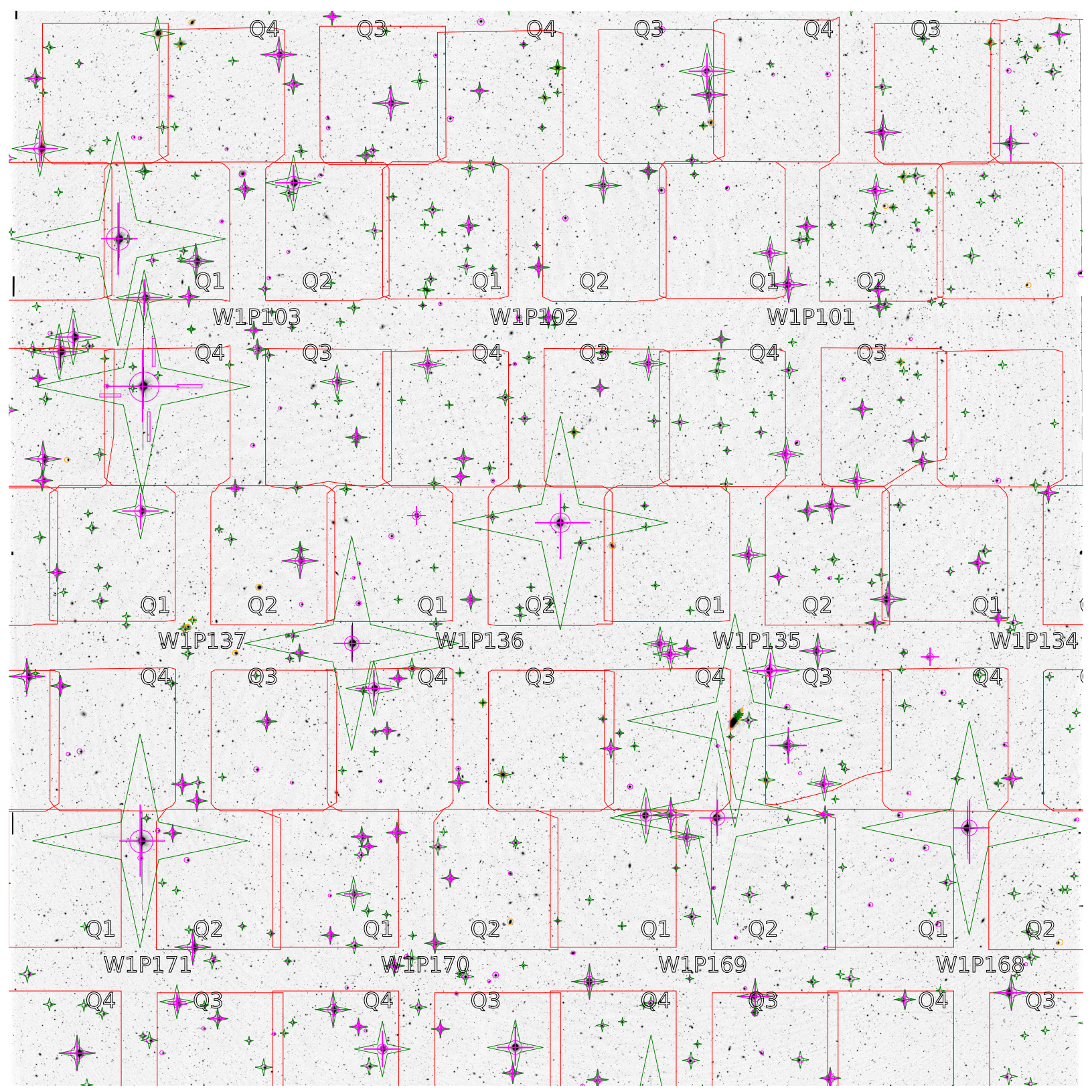

Fig. 9. Visual display of the masks developed for VIPERS, inside a $1 \mathrm{deg}^{2}$ region of the survey. The new bright-star mask is marked by the magenta circles and cross patterns, while the original mask distributed by Terapix, based on the four-point star template, is shown in green; orange polygons are drawn around selected extended sources. The quadrants that make up the VIPERS pointings are plotted in red. In the background is the CFHTLS T0006 $\chi^{2}$ image of the field 020631-050800 produced by Terapix. Note the significant gain in usable sky obtained with the new VIPERS-specific mask.

template with a cross pattern. The angular size of the template is scaled based upon the magnitude of the star.

Our starting point for the bright star mask was the USNOB 1.0 catalogue (Monet et al. 2003), from which we selected a sample of stars with $B_{\text {Vega }}<17.5$. Using the full CFHTLS area $\left(130 \mathrm{deg}^{2}\right)$, we measured the mean source density in the photometric catalogue as a function of distance from a bright star. We used the density profile to calibrate a size-magnitude relation for the stellar halo. We derived the following relations for the star magnitude $B$ and the halo radius $R$ in arcminutes:

$$
\begin{aligned}
& B<15.19: \log _{10}(R)=-2.60 \log _{10}(B)+2.33 \\
& B \geq 15.19: \log _{10}(R)=-6.55 \log _{10}(B)+6.99 .
\end{aligned}
$$

For stars brighter than $B=17$ we include a cross pattern to cover the diffraction spikes. For the brightest $\sim 200$ stars with $B<11$, we inspected the $\chi^{2}$ image (see Szalay et al. 1999) and adjusted the masks individually. The USNO B catalogue includes a number of extended sources that in many cases have multiple entries. We cross-checked the catalogue against the 2MASS Extended Source Catalogue to remove duplicates. A zoom into the W1 field, showing the various masks, is displayed in Fig. 9.

Although significant attention was given to constructing a homogeneous imaging survey in five bands, a handful of patches exist within the W1 field that have degraded photometric quality in one band. These regions were identified based upon high values of the photometric redshift $\chi^{2}$. We include these regions as rectangular patches in the photometric mask, visible in Fig. 9. No such regions were identified in the W4 field. 

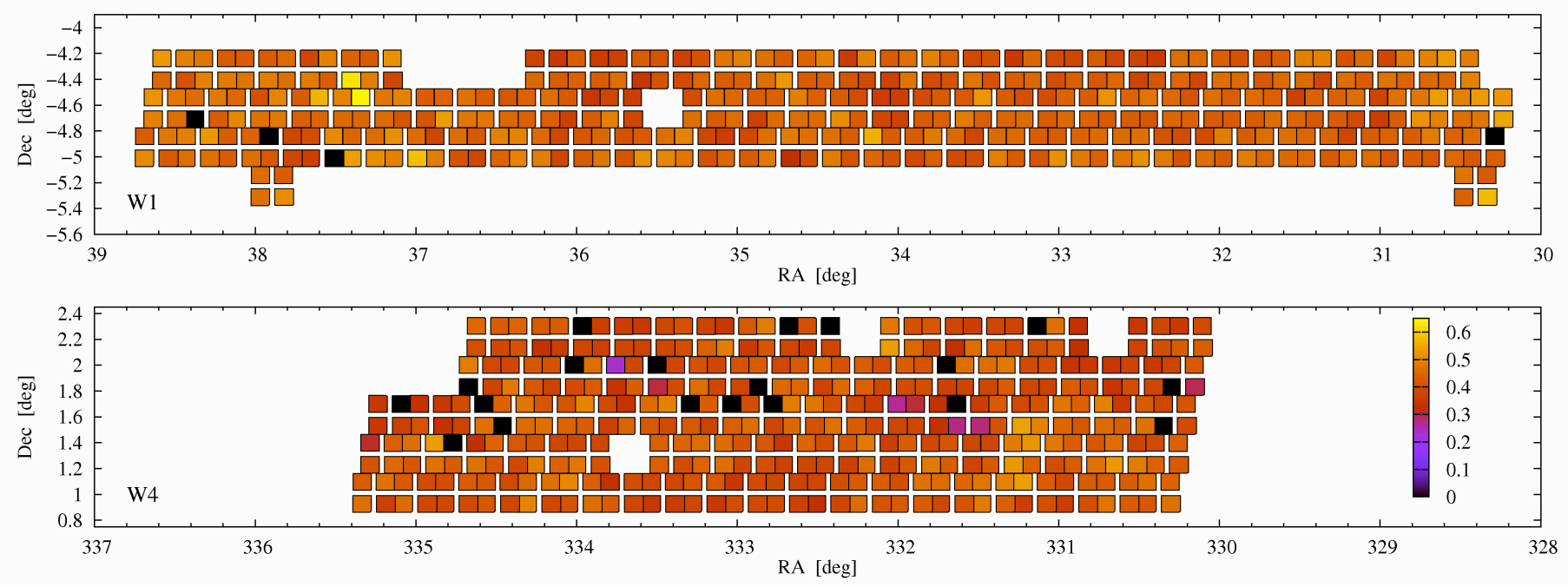

Fig. 10. Layout on the sky of all pointings included in the PDR-1 catalogue, for the two fields W1 and W4. Each of the four quadrants composing the pointings is shown and colour-coded according to the specific target sampling rate (TSR) over its area. The TSR is simply the ratio of the number of targeted galaxies over the number of potential targets. As shown, the average TSR is around 40\%. Black quadrants correspond to a failure in the insertion of the mask for that specific quadrant and the consequent loss of all data.

\subsection{Spectroscopic mask and weights}

Although the general layout of VIMOS is well known, the precise geometry of each quadrant's observations need to be specified carefully, in order to perform precise clustering measurements with the VIPERS data. Although it happens rarely, a quadrant may be partly vignetted by the guide probe arm, in those cases in which no better located guide star could be found. In addition, the accurate size and geometry of each quadrant was changed between the pre- and the post-refurbishment data (i.e. from mid-2010 on), due to the dismounting of the instrument and the technical features of the new CCDs. We had therefore to build our own extra mask of the spectroscopic data, accounting for all these aspects at any given point on the sky covered by the survey.

The masks for the $\mathrm{W} 1$ and $\mathrm{W} 4$ data were constructed from the pre-imaging observations by running an image analysis routine that identifies "good" regions. First, a polygon is defined that traces the edge of the image. The mean and variance of the pixels are computed in small patches at the vertices of the polygon. These measurements are compared to the statistics at the centre of the image. The vertices of the polygon are then iteratively moved inward toward the centre until the statistics along the boundary are within an acceptable range. The boundary that results from this algorithm is used as the basis for the field geometry. The polygon is next simplified to reduce the vertex count: short segments that are nearly co-linear are replaced by long segments. The WCS information in the fits header is used to convert from pixel coordinates to sky coordinates. Each mask was then examined by eye. Features due to stars at the edge of an image were removed, wiggly segments were straightened and artefacts due to moon reflections were corrected. The red lines in Fig. 9 show the detailed borders of the VIMOS quadrants, describing the spectroscopic mask.

Before scientific analyses can be performed on the observed data, knowledge of two more selection functions (angular masks) is needed, i.e. the TSR and SSR mentioned earlier. The variation of the TSR as a function of quadrant is shown in Fig. 10, reflecting the intrinsic fluctuations in the number density of galaxies as a function of position on the sky. Thanks to the adopted strategy (i.e. having discarded through the colour selection almost half of the magnitude-limited sample lying at $z<0.5$ ), the average TSR of VIPERS is $>40 \%$, a fairly high value that represents one of the specific important features of VIPERS. This can also be appreciated in Fig. 12 (bottom histogram), where we plot the TSR integrated over the whole survey, as a function of galaxy magnitude. Note how the TSR is substantially independent of the target magnitude.

Similarly, the SSR corresponding to measuring a reliable redshift (flag $=2,3,4,9$ ) over the VIMOS quadrants is shown in Fig. 11. Here one can appreciate how for the majority of the survey area we have $\operatorname{SSR}_{2,3,4,9}>80 \%$. A few observations under problematic conditions (either technical or atmospheric) are clearly marked out by the brown and purple rectangles. In Fig. 12 (top histograms) we also plot the SSR integrated over the whole survey as a function of the target magnitude, as detailed in the caption. As one would expect, faintest galaxies are harder to measure: at the very limit of the VIPERS survey $\left(22<i_{A B}<22.5\right)$, a redshift is delivered for $\sim 90 \%$ of the galaxies; a reliable redshift is obtained for as many as $\sim 75 \%$ of the targeted galaxies. The SSR shown as a colour scale in Fig. 11 corresponds specifically to the latter case, integrated over all magnitudes.

Through the observed dependence on apparent magnitude, one would expect in general a dependence of the SSR on redshift, SSR $(\operatorname{mag}(z))$. An explicit dependence may in principle also arise, however, due to the varying ability to identify spectral lines in regions of higher noise (e.g. where the "forest" of sky lines is stronger, at $\lambda>8000 \AA$ ). In practice, an estimate of the full $\operatorname{SSR}(\mathrm{mag}, z)$ can be obtained by using photometric redshifts for the unobserved targets. In this approach it is assumed that the quadrant-dependence $\operatorname{SSR}(Q)$ can be separated (i.e. only contributes a scaling factor) from the $\operatorname{SSR}(m a g, z)$. Such SSR $(\operatorname{mag}, z)$ has been used, e.g., in the computation of the luminosity and mass functions (Davidzon et al. 2013; Fritz et al. 2014).

For some specific analyses one may have to further correct for angular variations of the TSR and SSR on scales smaller than those of a single quadrant. For the TSR, this is the case of clustering measurements, to account for the "proximity bias" arising from the combination of the finite size of slits and spectra and 

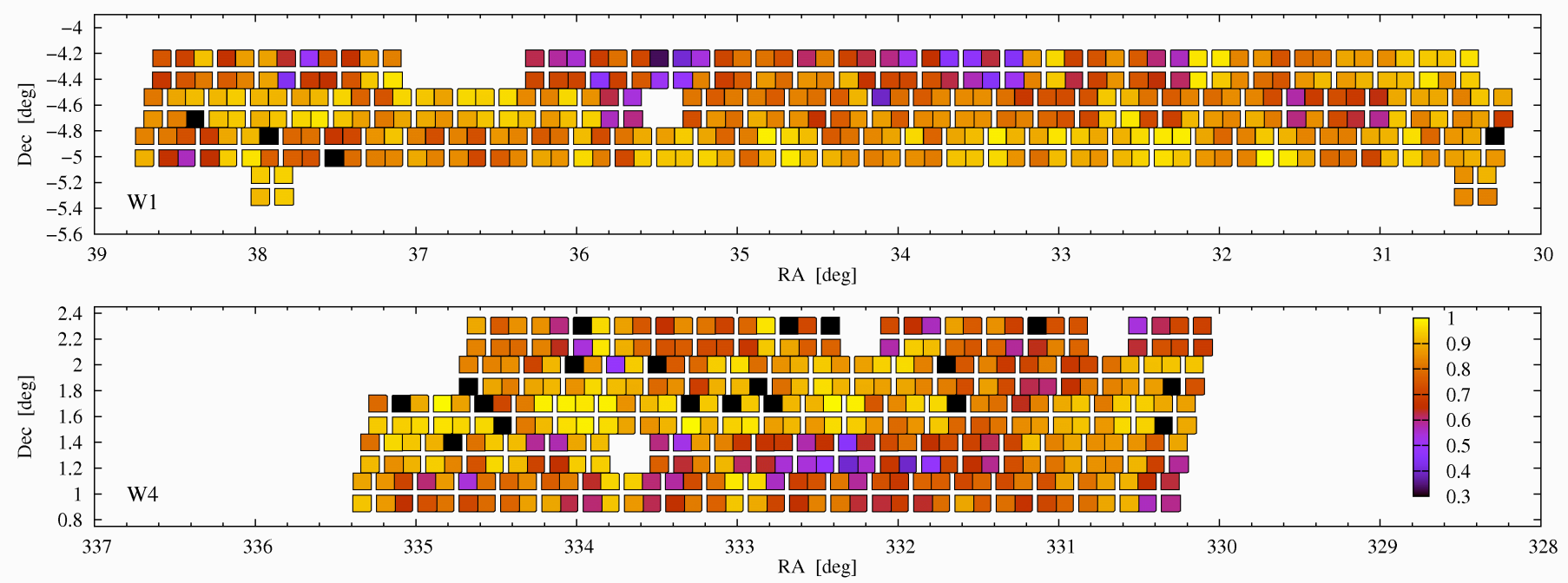

Fig. 11. Same as Fig. 10, but now with the colour-coding measuring the spectroscopic success rate (SSR), i.e. the ratio of the number of reliably measured redshifts (i.e. quality flag $\geq 2$ ) to the number of targeted galaxies. Also in this figure a few problematic areas emerge: purple and brown quadrants correspond to regions in which the fraction of successful measurements is, respectively, below $50 \%$ and $70 \%$. As can be seen, for the majority of quadrants the success rate is larger than $80 \%$

the single-pass strategy, which affects angular correlations below 0.05 degrees (de la Torre et al. 2013). Variations of the SSR on sub-quadrant scales are less likely, but could arise, for example, in case of optical field distortions that would produce an imperfect centring of the slits on the objects at the corner of the quadrants.

More discussion on such details is presented in the paper accompanying the PDR-1 catalogue (Garilli et al. 2014).

\section{Results and perspectives}

Experience with the first half of the VIPERS dataset fully confirms the expected general performance and science potential of the survey. As shown here, the average quality of the redshifts is as expected, with typical redshift measurement errors that are even better than in previous similar surveys with VIMOS. Figure 13 shows the redshift distribution of the data collected so far in the two fields. The combination of the two fields provides an impressively smooth distribution, averaging over local structure. As discussed earlier, the survey is complete beyond $z=0.6$, with a transition region at $0.4<z<0.6$ produced by the colourcolour selection. A substantial tail of galaxies out to $z=1.4$ is also apparent. This redshift range benefits particularly strongly from the increased sensitivity and lack of substantial fringing with the refurbished VIMOS CCDs, allowing a clearer detection of the [OII] 3727 line or the $4000 \AA$ break beyond $8000 \AA$.

The most striking result from this first significant set of VIPERS observations is provided by the new maps of the 3D galaxy distribution in the range $0.5<z<1.2$, which we show in the cone diagrams of Fig. 14. As demonstrated by these plots, VIPERS provides an unpredecented combination of overall size and detailed sampling, yielding a representative picture of the overall galaxy population and large-scale structure when the Universe was about half its current age. A direct comparison of VIPERS with local surveys, in terms of size and redshift, is shown in Fig. 15. Here the VIPERS redshift data are plotted together with those from the SDSS-Main and SDSS-LRG surveys. The fidelity with which structure can be seen in VIPERS (covering linear scales $\sim \mathrm{Gpc}$ ) is comparable, at high redshifts, to that of SDSS-Main at $z<0.1$, while the lower density of the LRG sample conveys little visual impression of significant structure.

New statistical measurements of clustering are being obtained with these results. Moreover, the rich and high-quality set of ancillary photometric data, combined with the distance information, is allowing us to compute the key metadata (SED, luminosities, stellar masses) for quantifying the connection between galaxy properties and the surrounding structure at these early epochs. An example of the power of correlating galaxy properties with the surrounding large-scale structure is provided by Fig. 16, which represents a zoom into part of the W1 VIPERS volume. Here galaxies have been coloured according to their rest-frame $U-B$ colour, providing in this way obvious evidence that the present-day colour-density relation had already been established at these redshifts.

The scientific investigations of the VIPERS Team using this rich dataset have focused so far on a series of goals, which we briefly list here:

- To measure the growth of structure between $z=1.2$ and 0.5 , by modelling the anisotropy of clustering (de la Torre et al. 2013). The initial application is to the galaxy population treated as a whole, but the high sampling and good spectroscopic completeness means that we will be able to exploit the use of multiple populations to reduce statistical and systematic errors in this measurement.

- To measure in detail the clustering of galaxies on small/intermediate scales at $0.5<z<1$, quantifying its dependence on luminosity and stellar mass (Marulli et al. 2013). The final goal here is to describe the relation between baryons and dark matter, measuring the evolution of the galaxy HOD (de la Torre et al., in prep.).

- To measure the power spectrum of the galaxy distribution $P(k, z)$ over $0.5<z 1.2$ (Rota et al., in prep.), constraining cosmological parameters like the matter density parameter (Bel et al. 2014), and the neutrino mass and number of species (Granett et al. 2012; Xia et al. 2012).

- To measure the luminosity and stellar mass functions to high statistical accuracy at $0.5<z<1$, in particular at the bright/massive end (Davidzon et al. 2013). 


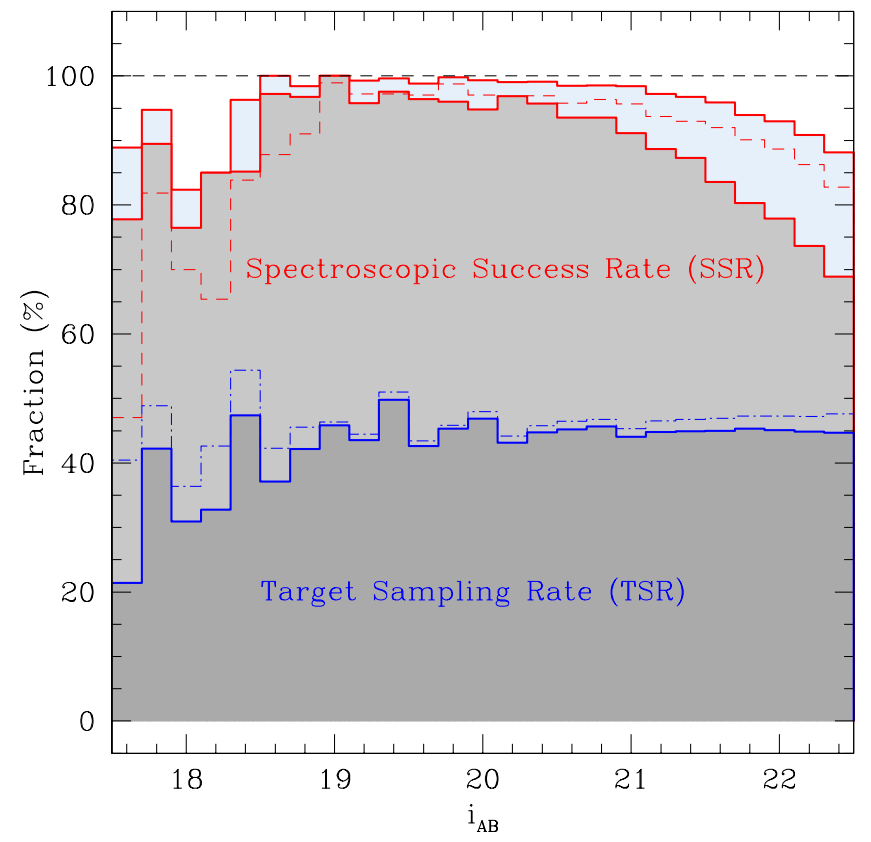

Fig. 12. Target sampling rate (TSR, lower histograms) and spectroscopic success rate (SSR, upper histograms), as a function of galaxy magnitudes. TSR and SSR are defined as in the text, but here the TSR is shown for two cases: the solid line corresponds to considering as targets only those objects for which an actual spectrum is detected in the corresponding slit; the dot-dashed line considers instead all placed slits, independently from having an actual spectrum detected (which is what we usually call TSR and is shown quadrant-by-quadrant in Fig. 10). This latter plot shows that slits are placed on targets in a way that is substantially independent of galaxy magnitudes, with a slight tendency to favour the fainter objects. The solid histogram shows on one side that about $2 \%$ of the targets are not detected, on average, for $i_{A B}>20$, with this fraction increasing from $0.5 \%$ to $4 \%$ when going from $i_{A B}=20$ to 22.5. On the other hand, it also shows a difficulty in detecting and extracting the spectra at the bright end $\left(i_{A B}<19\right)$. We understand this as due to the brighter (larger) objects filling completely the short slits adopted, thus making their detection in the 2D spectrum more difficult (see also Garilli et al. 2014). For the SSR (top curves), the two solid histograms give the success rate with respect to the detected targets, but using respectively all measured redshifts (flag $\geq 1$, lighter shading) or only the "reliable" redshifts (flag $\geq 2$, medium shading). The shortdashed line gives instead the flag $\geq 1$ SSR, but referred to all targeted galaxies. The SSR drops for the very bright objects, due to the reason discussed for the TSR. See Garilli et al. (2014) for more discussion on using these quantities in statistical calculations.

- More generally, to make a full characterisation of the evolution of galaxies over this important range of redshifts, in terms of the distributions of other fundamental properties like colours, spectral types and star-formation rates (Fritz et al. 2014). This will also benefits of the high quality of the CFHTLS observations in terms of average PSF to push simple morphological estimations (bulge/disk decomposition) to the higher redshifts explored by VIPERS (Krywult et al., in prep.).

- To measure higher-order clustering statistics at this early epoch, where mass fluctuations are closer to the linear regime, measuring the moments of the galaxy distribution (Cappi et al., in prep.) and the evolution and nonlinearity of galaxy biasing (Di Porto et al., in prep.).

- To construct a large and well defined sample of optically selected groups and clusters at at $0.5<z<1$, to investigate the properties of these systems and in particular the evolution of galaxies in different environments (Iovino et al., in prep.).

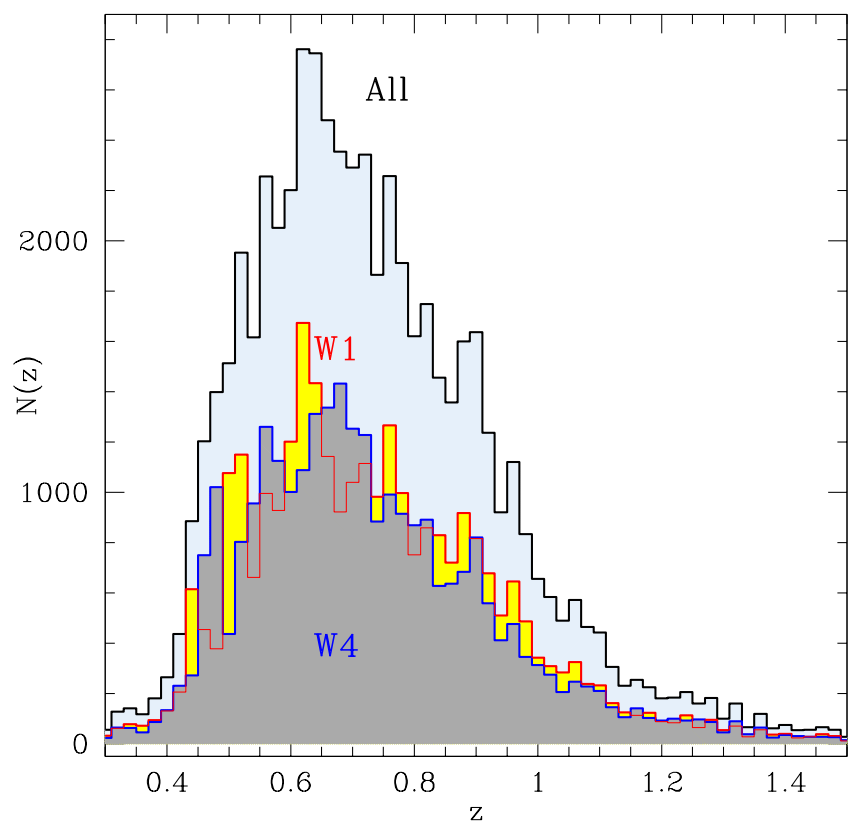

Fig. 13. Galaxy redshift distribution from the full VIPERS PDR-1 catalogue (solid black line), and separately within the W1 and W4 fields (solid red and blue lines, respectively). All measured redshifts (flag $=1$ and above) have been plotted here. The redshift histogram restricted to only the most reliable redshifts (flag $\geq 2$ ) does not show significant differences.

- To reconstruct the density field over a large volume and dynamic range at $0.5<z<1$, properly accounting for the specificity of the VIPERS footprint and sampling (Cucciati et al. 2014).

- Using this density field, to produce an order-of-magnitude improvement in our knowledge of crucial relationships between galaxy properties and their environment, as colours (Cucciati et al., in prep.), and stellar masses (Davidzon et al., in prep.).

- To construct a massive spectroscopic and multi-band photometric database, with automatic spectral classifications through SED-fitting, Principal Component Analysis (Marchetti et al. 2013) and other techniques, such as supervised learning algorithm methods (Małek et al. 2013).

- To cross-correlate the detailed 3D maps of the galaxy distribution with the dark-matter maps reconstructed using weak lensing from the CFHTLS high-quality images.

- To measure the faint end of the AGN luminosity function and their correlation with large-scale structure, through a dedicated sub-sample.

This is a substantial list of what should prove to be exciting developments, representing a major advance in our knowledge of the structure in the Universe around redshift unity. But all these applications should benefit from more detailed investigation, and there are many fruitful topics beyond those listed above. We hope, and expect, that VIPERS will follow in the path of the major low-redshift surveys in generating many more important papers from open use of the public data. We therefore encourage readers to start using the PDR-1 data release ${ }^{4}$, described in detail in its accompanying paper (Garilli et al. 2014). This should serve to increase anticipation for what may be achieved with the final VIPERS dataset, which, given the current statistics, is expected to include around 90000 redshifts.

4 Available at http://vipers.inaf.it 

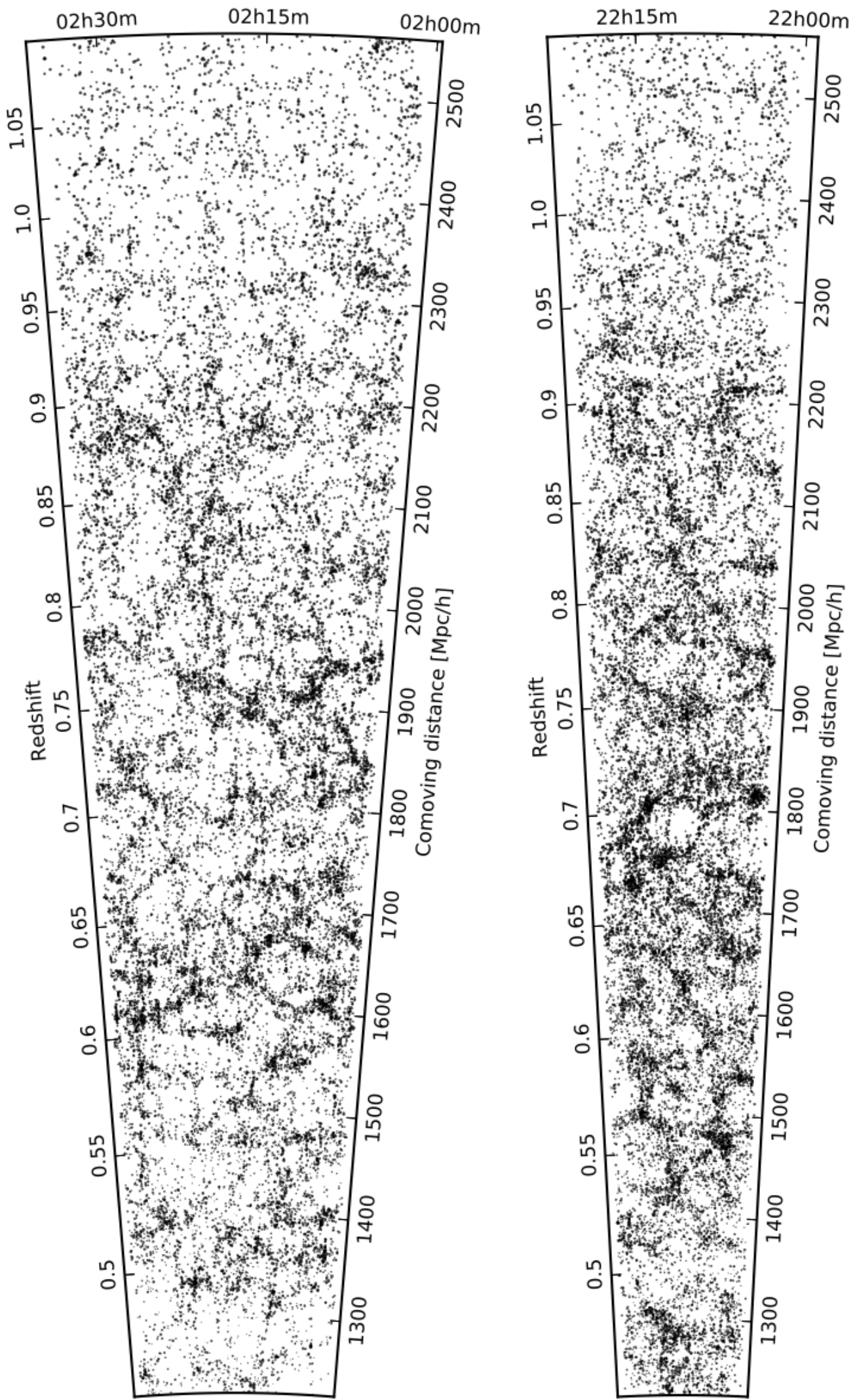

Fig. 14. Large-scale galaxy distribution at $0.45<z<1.1$ unveiled by the VIPERS PDR-1 catalogue in the CFHTLS W1 and W4 fields (left and right respectively), currently including $\sim 55000$ redshifts. Galaxy positions are projected along the declination direction, where the width is $\simeq 1^{\circ}$ for $\mathrm{W} 1$ and $\simeq 1.5^{\circ}$ for $\mathrm{W} 4$. Note the high-resolution sampling of large-scale structure in VIPERS, comparable to that of SDSS Main and $2 \mathrm{dFGRS}$ at $z<0.2$. 


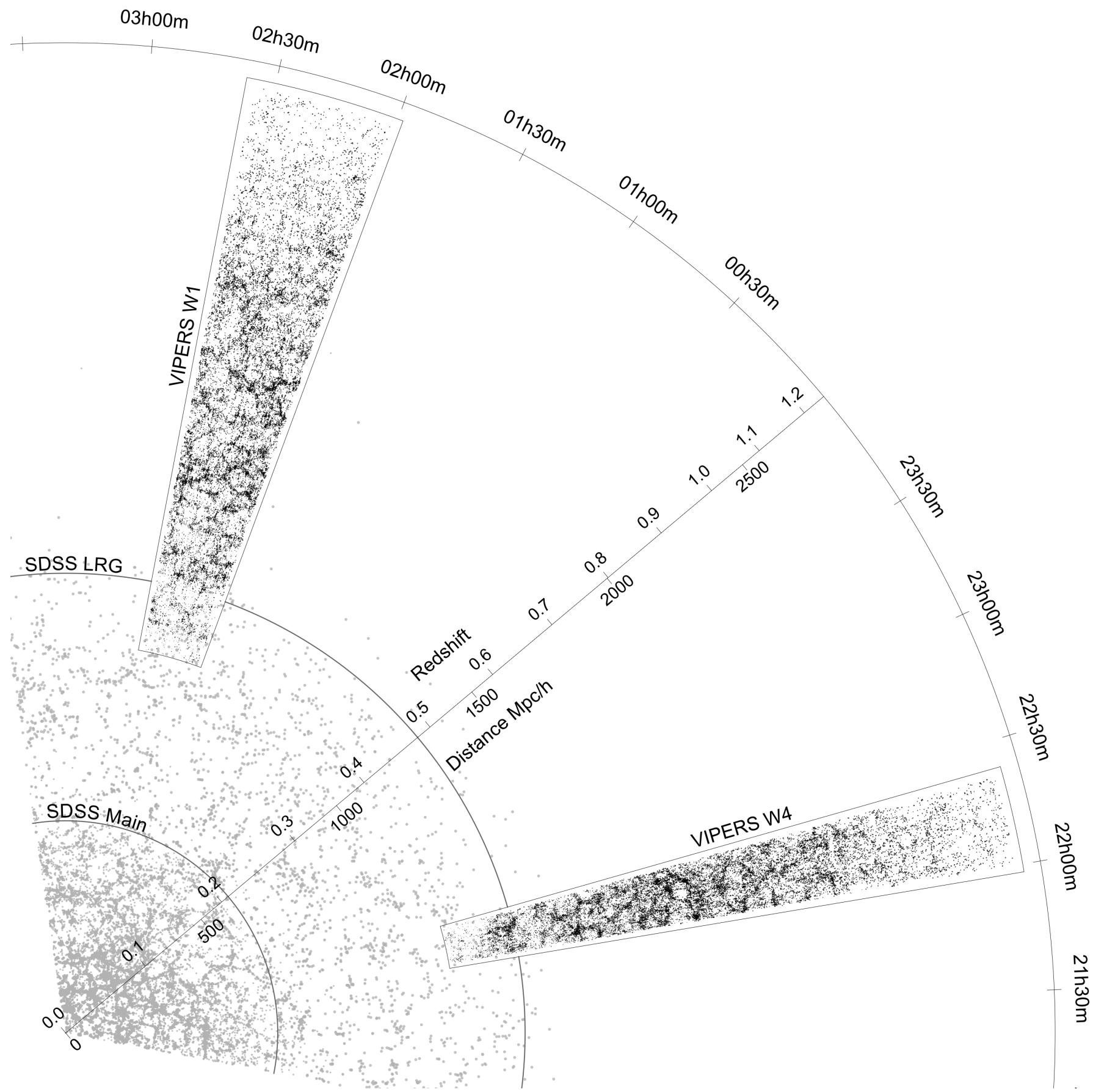

Fig. 15. Putting VIPERS in perspective. This plot shows the complementarity of the $0.5<z<1.5$ regions probed by the two VIPERS deep fields, and the SDSS main and LRG samples at lower redshift (for which a 4-degree-think slice is shown). The LRG samples are excellent statistical probes on the largest scales, but (by design) they fail to register the details of the underlying nonlinear structure, which is clearly exposed by VIPERS.

Acknowledgements. We acknowledge the crucial contribution of the ESO staff for the management of service observations. In particular, we are deeply grateful to M. Hilker for his constant help and support to this program. Italian participation to VIPERS has been funded by INAF through PRIN 2008 and 2010 programs. L.G., B.R.G. and J.B. acknowledge support from the European Research Council through the Darklight ERC Advanced Research Grant (\# 291521). O.L.F. acknowledges support from the European Research Council through the EARLY ERC Advanced Research Grant (\# 268107). Polish participants have been supported by the Polish Ministry of Science (grant N203 5129 38), the Polish-Swiss Astro Project (co-financed by a grant from Switzerland, through the Swiss Contribution to the enlarged European Union), the European Associated Laboratory Astrophysics Poland-France HECOLS and a Japan Society for the Promotion of Science (JSPS) Postdoctoral Fellowship for Foreign Researchers
(P11802). G.D.L. acknowledges financial support from the European Research Council under the European Community's Seventh Framework Programme (FP7/2007-2013)/ERC grant agreement No. 202781. W.J.P. and R.T. acknowledge financial support from the European Research Council under the European Community's Seventh Framework Programme (FP7/2007-2013)/ERC grant agreement No. 202686. W.J.P. is also grateful for support from the UK Science and Technology Facilities Council through the grant ST/I001204/1. E.B., F.M. and L.M. acknowledge support from grants ASI-INAF I/023/12/0 and PRIN MIUR 2010-2011. Y.M. acknowledges support from CNRS/INSU (Institut National des Sciences de l'Univers) and the Programme National Galaxies et Cosmologie (PNCG). C.M. is grateful for support from specific project funding of the Institut Universitaire de France and the LABEX OCEVU. 


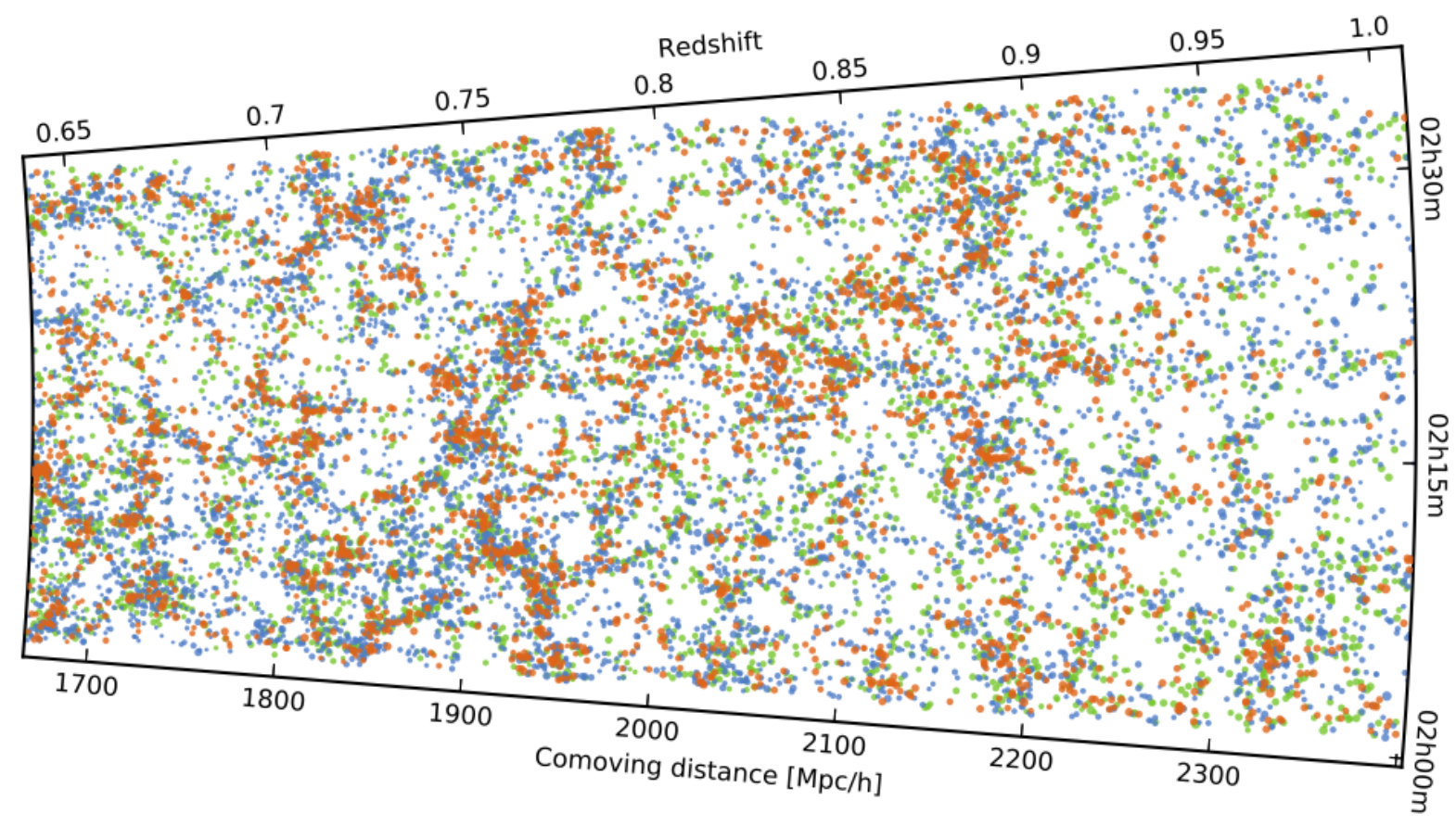

Fig. 16. Zoom on the galaxy distribution within the VIPERS W1 field, where now the additional dimension of galaxy rest-frame colours has been added. Galaxies are here marked in blue, green or reddish, depending on whether their $U-B$ rest-frame colour is respectively $<0.9$, between 0.9 and 1.2, or $>1.2$. Also in this case the size of the dots has been set proportionally to the $B$-band luminosity of the corresponding galaxy. The plot shows clearly that the colour-density relation for galaxies is already in place at these redshifts (Cucciati et al. 2006), with red early-type galaxies tracing the backbone of structure and blue/green star-forming objects filling the more peripheral lower-density regions. This picture gives an example of the potential of VIPERS for studying the clustering of galaxies as a function of galaxy properties, over scales ranging from less than a Mpc to well above $100 \mathrm{Mpc}$.

\section{Appendix A: VIPERS star-galaxy separation}

The star/galaxy classification scheme developed to construct the VIPERS target sample benefits from the high-quality CFHTLS photometric data combined with the available spectroscopic information for a significant number of objects in both W1 and W4 provided by the VVDS Deep and Wide surveys. The CFHTLS photometric data are particularly suited for this operation. Having been designed for weak-lensing studies, they benefit of sub-arcsec seeing over most of the survey which makes identification of point sources much easier compared to other surveys. This is a significant asset of VIPERS and allows us to perform an accurate star/galaxy selection and in turn make efficient use of telescope time. This is particularly important as in a purely magnitude limited sample of objects at $i_{A B}<22.5$ the fraction of stars can be larger than $30 \%$ (as it is the case in the W4 field).

A key ingredient in identifying the optimal selection criteria for star-galaxy separation is provided by the two large and complete pre-existing spectroscopic samples in VIPERS fields, i.e. VVDS-Deep (Le Fèvre et al. 2005) and VVDSWide (Garilli et al. 2008). VVDS-Deep provides redshifts for more than 10000 galaxies, AGNs and stars to $i_{A B}=24$, over $\mathrm{a} \sim 0.5 \mathrm{deg}^{2}$ area in W1. The F22 field of VVDS-Wide, instead, includes spectra over $4 \mathrm{deg}^{2}$ for 11200 galaxies and $\sim 7000$ stars to $i=22.5$, in W4. These two VVDS samples are purely magnitude-limited surveys. They represent therefore an ideal control sample to test the completeness and contamination of any selection criterion. Here we use only the most secure unambiguous spectra and restrict the Deep and Wide VVDS catalogues to only flag 3 and 4 objects (defined in a scheme analogous to that described in Sect. 5.2).

\section{A.1. Methods and tests}

The method adopted to classify stars and galaxies for VIPERS combines knowledge of the object size, provided by the halflight radius $r_{\mathrm{h}}$ (i.e. the radius containing half of the object's flux), with that of its reconstructed spectral energy distribution (SED), obtained through template fitting of the available fiveband photometry.

The excellent image quality of the CFHTLS data suggests that at the VIPERS magnitudes the object size $r_{\mathrm{h}}$ should provide the prime way to distinguish stars from galaxies. Figure A.1 plots the magnitude and the size of a complete set of spectroscopically identified stars and galaxies from the VVDS-Wide survey (Garilli et al. 2008) which overlaps tile \#5 of the VIPERS W4 area. The sharply defined locus occupied by stars (blue asterisks), defines the typical size of a point-like source in this tile which depends on the tile seeing (note that the few red points appearing over the stellar locus for $i<21$ correspond to AGN). In order to characterise the intrinsic point spread function (PSF) of each tile, we select objects with $17.5<i<21$ where stars are dominant and fit a Gaussian to the $r_{\mathrm{h}}$ distribution. The statistical distribution of stellar sizes within a specific tile in this way can be described in terms of its mean $\left(\mu_{\mathrm{rh}}\right)$ and standard deviation $\left(\sigma_{\mathrm{rh}}\right)$. Looking at Fig. A.1, it is natural to define as stars objects with $r_{\mathrm{h}}<\mu_{\mathrm{rh}}+3 \sigma_{\mathrm{rh}}$. Even excluding AGN interlopers, however, one sees that for magnitudes fainter than $i \simeq 21$ a number of small galaxies exist which would be mistaken as stars by purely geometrical criteria.

To recover galaxies at the faintest limit and increase completeness of the galaxy sample we add therefore the type information provided by the object SED. This is obtained by fitting the five-band CFHTLS photometry with the Le Phare 


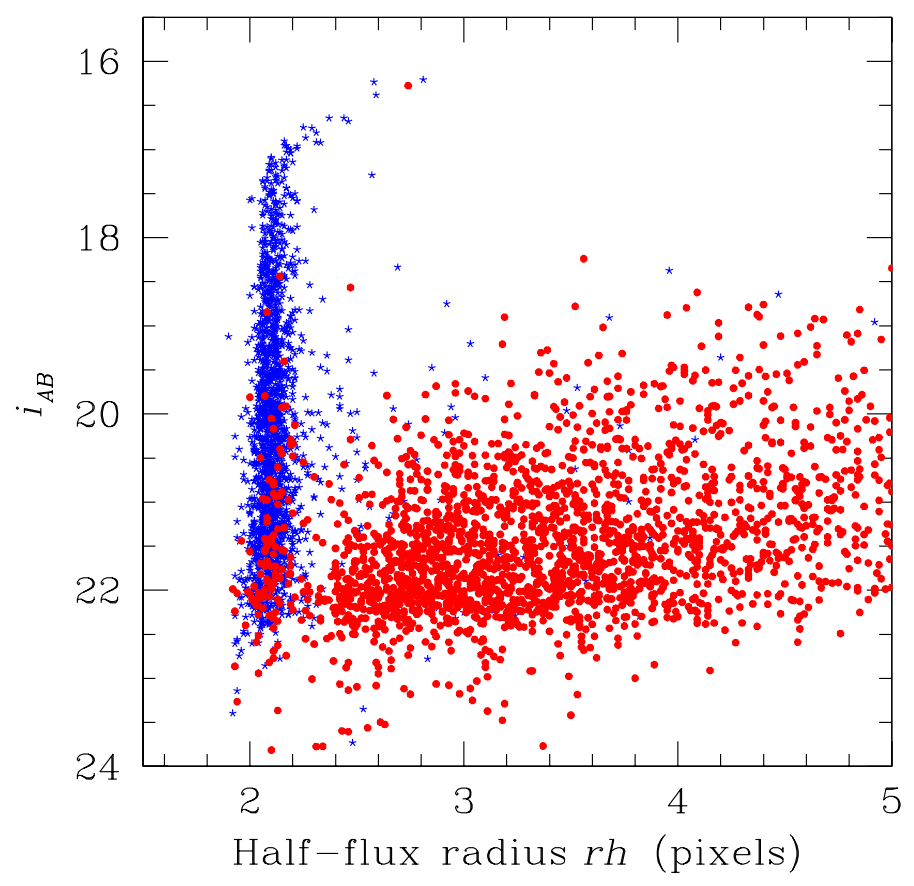

Fig. A.1. Object size, measured by the radius enclosing half of the object's flux, $r_{\mathrm{h}}$, with the $i$ magnitude. This is done here for a complete set of spectroscopically identified stars and galaxies from the VVDS-Wide survey (Garilli et al. 2008). All objects belong to tile \#5, in the overlapping region with the VIPERS W4 area, and are therefore characterised by a uniform seeing (see text). Stars are plotted as blue asterisks and galaxies as red points. The locus of point-like sources is well defined, suggesting a clear strategy for star-galaxy separation as discussed in the text. The few red points lying within the stellar locus at bright magnitudes $\left(i_{A B}<21\right)$ correspond to (point-like) AGNs.

photometric redshift code. Among a library of SEDs, the bestfitting $\chi^{2}$ is identified for both galaxy $\left(\chi_{\text {gal }}^{2}\right)$ and stellar $\left(\chi_{\text {star }}^{2}\right)$ templates. An object is then classified as a galaxy (star) if $\chi_{\text {gal }}^{2}$ is smaller (larger) than $\chi_{\text {star }}^{2}$. The corresponding limitation of this technique is that with the available optical (ugriz) bands, there is a degeneracy in colour of some stars and galaxies which would result in significant stellar contamination if only this method is used. This is shown by the plots of Fig. A.2. For this reason the final VIPERS criteria have been defined as a combination of these two methods.

To quantify the performances of our different selection criteria, we first define incompleteness and contamination. Let us define $N_{\text {est }}$ the number of objects classified as galaxies by a given method; this contains both real galaxies $N_{\text {est-true }}$ and stars misclassified as galaxies $N_{\text {est-fake }}$, such that $N_{\text {est }}=N_{\text {est-true }}+N_{\text {est-fake }}$. Let us also call $N_{\text {true }}$ the total number of galaxies in the sample. Using our VVDS control samples we know all these contributions and can thus estimate the intrinsic theoretical incompleteness of a selection method as

Inc $=\frac{\left(N_{\text {true }}-N_{\text {est-true }}\right)}{N_{\text {true }}}$.

Similarly, the theoretical sample contamination is

$C n t=\frac{N_{\text {est-fake }}}{N_{\text {true }}}$.

Clearly, in real observations we only know $N_{\text {est }}=N_{\text {est-true }}+$ $N_{\text {est-fake, }}$, and we can only define incompleteness and contamination with respect to the recovered sample of galaxies. For testing these methods with the VVDS data, however, here we have

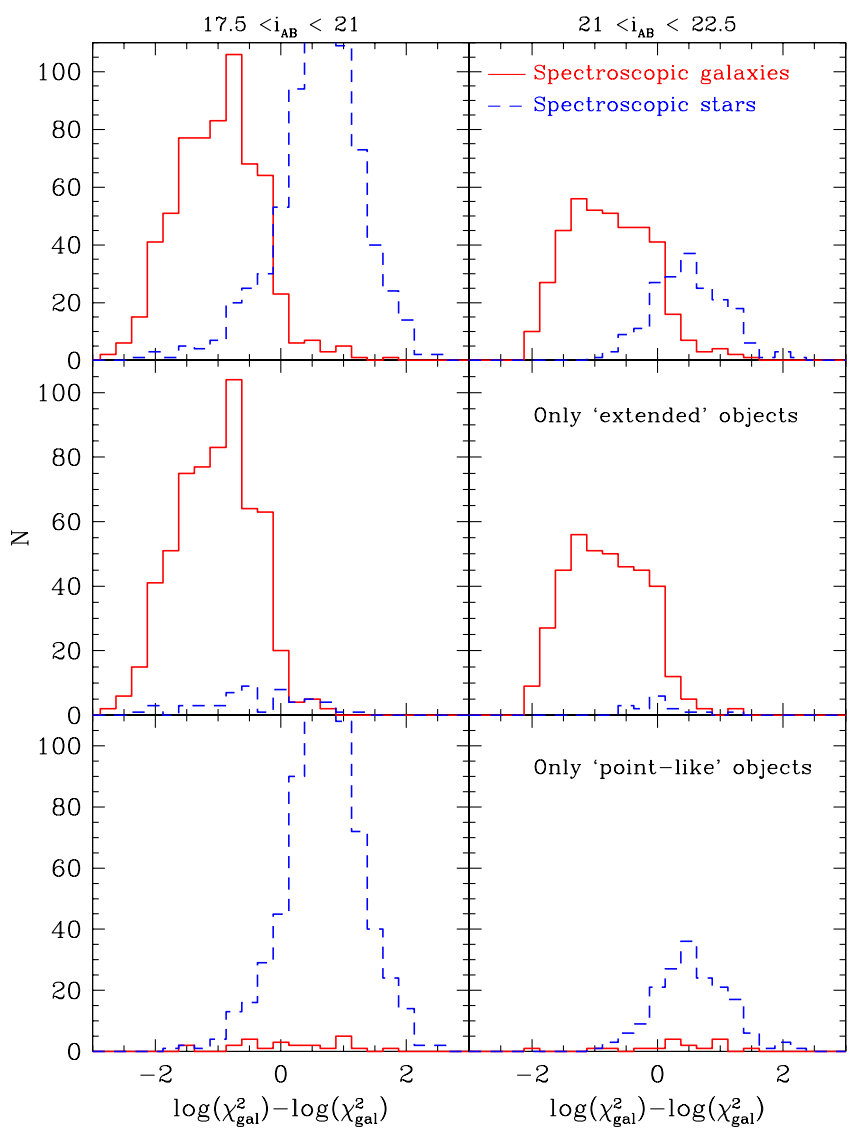

Fig. A.2. Distribution of $\log \left(\chi_{\text {star }}^{2}\right)-\log \left(\chi_{\text {gal }}^{2}\right)$ for spectroscopically confirmed stars (dashed histograms) and galaxies (solid histograms) for the VVDS-Wide spectroscopic sample in W4. The sample is split into a bright and faint sample, corresponding to the split used to classify VIPERS galaxies. Ideally, one would expect that all galaxies have $\chi_{\text {star }}^{2}-\chi_{\text {gal }}^{2}<0$, while stars are confined to positive values. However, as can be seen, tails of both populations overlap each other. Top: no selection is applied on the half-flux radius $r_{\mathrm{h}}$. Middle: only objects with $r_{h} \geq \mu_{\mathrm{rh}}+3 \sigma_{\mathrm{rh}}$ are considered (i.e. "geometric" galaxies). Bottom: only objects with $r_{h}<\mu_{\mathrm{rh}}+3 \sigma_{\mathrm{rh}}$ are considered (i.e. "geometric" stars).

preferred to work with the intrinsic expected quantities defined above.

After significant experimentation, the VIPERS stars-galaxy separation has been defined through the following combination of the two methods discussed earlier:

1. At $i<21$, stars are defined to be simply objects with $r_{\mathrm{h}}<$ $\mu_{\mathrm{rh}}+3 \sigma_{\mathrm{rh}}$. Galaxies are the complementary class.

2. At $i \geq 21$, stars are defined as having $r_{h}<\mu_{\mathrm{rh}}+3 \sigma_{\mathrm{rh}}$, but requiring in addition that $\log \left(\chi_{\text {star }}^{2}\right)<\log \left(\chi_{\text {gal }}^{2}\right)+1$. In this way, small-sized faint galaxies (i.e. objects for which $\log \left(\chi_{\text {star }}^{2}\right) \geq \log \left(\chi_{\text {gal }}^{2}\right)+1$ OR $\left.r_{h} \geq \mu_{\text {rh }}+3 \sigma_{\text {rh }}\right)$ are added to the sample thus increasing its completeness.

Applying this combination to the VVDS-Wide and VVDS-Deep test samples, we obtain the completeness Inc and contamination Cnt levels that are summarised in Table A.1. Within the limitations of the sample sizes, the figures in this table should represent a good indication of the estimated percentages expected in actual VIPERS data. The contamination level is the only one that can be checked directly using the actual observed data, to verify these predictions on a much largers sample. Considering the PDR-1 data, the outcome is extremely encouraging. Together with the 53608 confirmed galaxy spectra, the data composing 
Table A.1. Incompleteness and contamination of the VIPERS galaxy sample expected from the star-galaxy separation process, estimated by applying the final criteria discussed in the text to the VVDS Deep and Wide complete catalogues to $i_{A B}=22.5$.

\begin{tabular}{ccc}
\hline \hline Field & Inc & $C n t$ \\
\hline W1 (VVDS-Deep) & $2.07 \%(2.13) \%$ & $0.87 \%(0.27 \%)$ \\
W4 (VVDS-Wide) & $0.96 \%(0.64 \%)$ & $6.59 \%(8.24 \%)$ \\
\hline
\end{tabular}

Notes. The values in parentheses give the values corresponding to galaxies colour-selected to be at $z>0.5$, i.e. that would be part of the actual VIPERS target (Sect. 4).

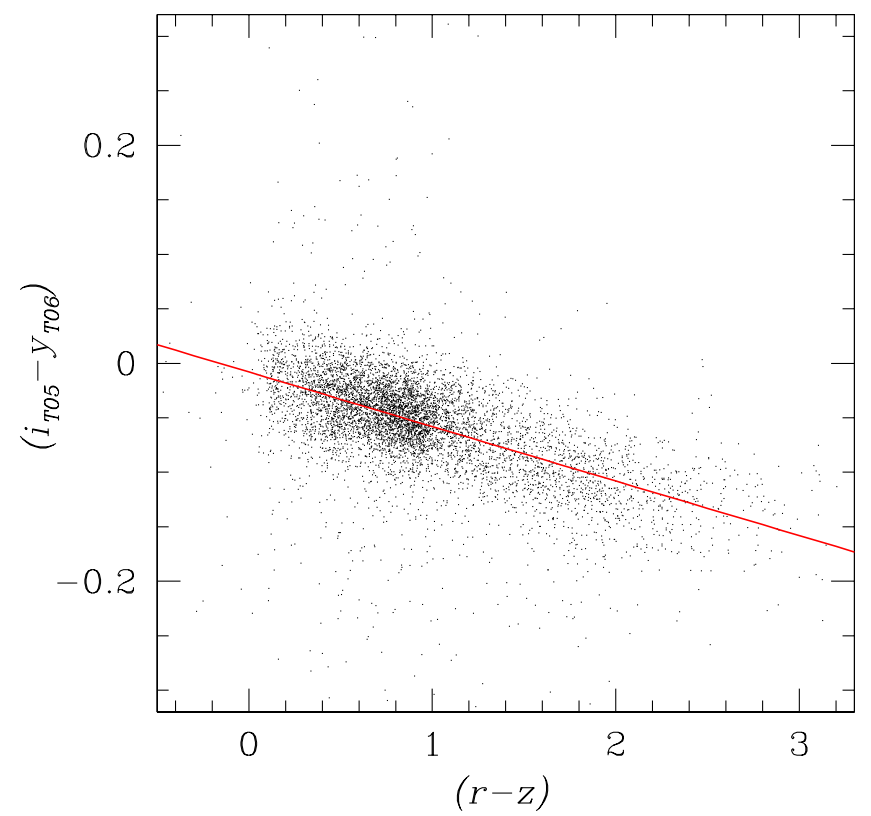

Fig. B.1. Colour transformation between the $i$-band magnitudes of objects in tile 022929-060400, as measured in the CFHTLS T0006 and T0005 catalogues using the original $i^{*}$ filter and its replacement (called $y$ or $i 2$, see text).

the PDR-1 catalogue have yelded also a set of 1750 stars that had been erroneously classified as galaxies and thus observed. This is what we called $N_{\text {est-fake }}$ in our scheme. To transform this precisely into a contamination $C n t$, we should know the incompleteness $I n c$ as to know the true expected number of galaxies in the sample. This cannot be obviously obtained from the observations. However, we can assume that the mean incompleteness is close to the value estimated from the VVDS samples and see whether the contamination agrees with the original expectation. Since the two samples from W1 and W4 composing the PDR-1 data set are very similar in number, the total incompleteness expected if we use the percentages estimated for the two fields in Table A. 1 is given by

$I n c_{\text {tot }} \simeq 1-\frac{(1-0.0213)+(1-0.0064)}{2}=1.39 \%$.

With this incompleteness, the average contamination in the current PDR-1 sample is

$C n t_{\mathrm{tot}}=\frac{1750}{53608(1+0.0139)}=3.22 \%$,

which on average is better than the mean value expected from the third column of Table A.1. If we do the same separately for the two fields $\mathrm{W} 1$ and $\mathrm{W} 4$, we obtain a contamination of $1.5 \%$
Table C.1. Cross-reference between the VIPERS numbering scheme and the corresponding CFHTLS tiles in the W1 field.

\begin{tabular}{cc}
\hline \hline W1 VIPERS Tile \# & CFHTLS name \\
\hline 01 & CFHTLS_W_ugriz_020241-060400_T0005 \\
02 & CFHTLS_W_ugriz_020631-060400_T0005 \\
03 & CFHTLS_W_ugriz_021021-060400_T0005 \\
04 & CFHTLS_W_ugriz_021410-060400_T0005 \\
05 & CFHTLS_W_ugriz_021800-060400_T0005 \\
06 & CFHTLS_W_ugriz_022150-060400_T0005 \\
07 & CFHTLS_W_ugriz_022539-060400_T0005 \\
08 & CFHTLS_W_ugriz_022929-060400_T0005 \\
09 & CFHTLS_W_ugriz_023319-060400_T0005 \\
10 & CFHTLS_W_ugriz_020241-050800_T0005 \\
11 & CFHTLS_W_ugriz_020631-050800_T0005 \\
12 & CFHTLS_W_ugriz_021021-050800_T0005 \\
13 & CFHTLS_W_ugriz_021410-050800_T0005 \\
14 & CFHTLS_W_ugriz_021800-050800_T0005 \\
15 & CFHTLS_W_ugriz_022150-050800_T0005 \\
16 & CFHTLS_W_ugriz_022539-050800_T0005 \\
17 & CFHTLS_W_ugriz_022929-050800_T0005 \\
18 & CFHTLS_W_ugriz_023319-050800_T0005 \\
19 & CFHTLS_W_ugriz_020241-041200_T0005 \\
20 & CFHTLS_W_ugriz_020631-041200_T0005 \\
21 & CFHTLS_W_ugriz_021021-041200_T0005 \\
22 & CFHTLS_W_ugriz_021410-041200_T0005 \\
23 & CFHTLS_W_ugriz_021800-041200_T0005 \\
24 & CFHTLS_W_ugriz_022150-041200_T0005 \\
25 & CFHTLS_W_ugriz_022539-041200_T0005 \\
26 & CFHTLS_W_ugriz_022929-041200_T0005 \\
27 & CFHTLS_W_ugriz_023319-041200_T0005 \\
\hline &
\end{tabular}

for $\mathrm{W} 1$ and $4.9 \%$ for $\mathrm{W} 4$, i.e. slightly higher than predicted for $\mathrm{W} 1$, but significantly smaller for W4.

\section{Appendix B: $i$-band filter transformation between T0005 and T0006}

As mentioned above, a few observations from the T0006 release that were needed for VIPERS to fill some missing "holes" in the original catalogue were in fact obtained with a different $i$-band filter with respect to the rest of T0005. The reason for this change was that the original $i$-band filter at CFHT (i . MP9701) broke in 2006 and was replaced. The new filter, i.MP9702, is called $y$ in TERAPIX documentation and sometimes also referred to as $i 2$. For the small number of objects in the VIPERS areas for which only the T0006 $y$-band measurement was available we derived a transformation using objects from the regions where both magnitudes are available. We considered one tile from the T0005 catalog,CFHTLS_W_ugriz_022929-060400_T0005.cat, and the corresponding T006 catalogue CFHTLS_W_ugryz_022929060400_T0006.catmask. These two lists were matched assuming that the T0005 data was based entirely on observations with the $i$ filter, and that the T0006 data was based entirely on observations with the $y$ filter. For bright and well measured objects $(18.0<i<21.0)$, we found a mean offset $\Delta_{i}=i_{\mathrm{T} 05}-y_{\mathrm{T} 06}=$ $-0.052 \pm 0.042 \mathrm{mag}$, and a good correlation between this offset and the observed $(r-z)$ colour, as shown in Fig. B.1, such that $\Delta_{i}=-0.008-0.050 *(r-z)$. Here the $(r-z)$ colour term accounts for the different response curve of the two filters. With this correction, all $i$-band magnitudes in the VIPERS catalogue should be considered as homogeneous. 
Table C.2. Cross-reference between the VIPERS numbering scheme and the corresponding CFHTLS tiles in the W4 field.

\begin{tabular}{cc}
\hline \hline W4 VIPERS Tile \# & \multicolumn{1}{c}{ CFHTLS name } \\
\hline 01 & CFHTLS_W_ugriz_220154+011900_T0005 \\
02 & CFHTLS_W_ugriz_220542+011900_T0005 \\
03 & CFHTLS_W_ugriz_220930+011900_T0005 \\
04 & CFHTLS_W_ugriz_221318+011900_T0005 \\
05 & CFHTLS_W_ugriz_221706+011900_T0005 \\
06 & CFHTLS_W_ugriz_220154+021500_T0005 \\
07 & CFHTLS_W_ugriz_222054+011900_T0005 \\
08 & CFHTLS_W_ugriz_220542+021500_T0005 \\
09 & CFHTLS_W_ugriz_220930+021500_T0005 \\
10 & CFHTLS_W_ugriz_221318+021500_T0005 \\
11 & CFHTLS_W_ugriz_221706+021500_T0005 \\
\hline
\end{tabular}

\section{Appendix C: CFHTLS-VIPERS tiles cross-numbering}

Tables C.1 and C.2 give the cross-reference between the CFHTLS tile names and the corresponding VIPERS internal numbering systems used throughout the survey selection process and in this paper.

\section{References}

Abazajian, K. N., Adelman-McCarthy, J. K., Agüeros, M. A., et al. 2009, ApJS, 182,543

Abbas, U., de la Torre, S., Le Fèvre, O., et al. 2010, MNRAS, 412, 825

Ahn, C. P., Alexandroff, R., Allende Prieto, C., et al. 2012, ApJS, 203, 21

Anderson, L., Aubourg, E., Bailey, S., et al. 2012, MNRAS, 427, 3435

Arnouts, S., \& Ilbert, O. 2011, LePHARE: Photometric Analysis for Redshift

Estimate, Astrophysics Source Code Library, ascl:1108.009

Bel, J., Marinoni, C., Granett, B. R., et al. 2014, A\&A, 563, A37

Bertin, E., \& Arnouts, S. 1996, A\&AS, 117, 393

Beutler, F., Blake, C., Colless, M., et al. 2012, MNRAS, 423, 3430

Blake, C., Brough, S., Colless, M., et al. 2011a, MNRAS, 415, 2876

Blake, C., Davis, T., Poole, G. B., et al. 2011b, MNRAS, 415, 2892

Bottini, D., Garilli, B., Maccagni, D., et al. 2005, PASP, 117, 996

Carroll, S. M., Duvvuri, V., Trodden, M., \& Turner, M. S. 2004, Phys. Rev. D, 70, 043528

Coil, A. L., Newman, J. A., Croton, D., et al. 2008, ApJ, 672, 153

Coil, A. L., Blanton, M. R., Burles, S. M., et al. 2011, ApJ, 741, 8

Cole, S., Percival, W. J., Peacock, J. A., et al. 2005, MNRAS, 362, 505

Colless, M., Dalton, G., Maddox, S., et al. 2001, MNRAS, 328, 1039

Cool, R. J., Moustakas, J., Blanton, M. R., et al. 2013, ApJ, 767, 118

Cooray, A., \& Sheth, R. 2002, Phys. Rep., 372, 1

Cucciati, O., Iovino, A., Marinoni, C., et al. 2006, A\&A, 458, 39

Cucciati, O., Granett, B. R., Branchini, E., et al. 2014, A\&A, 565, A67

Davidzon, I., Bolzonella, M., Coupon, J., et al. 2013, A\&A, 558, A23

Dawson, K. S., Schlegel, D. J., Ahn, C. P., et al. 2013, AJ, 145, 10

de la Torre, S., \& Guzzo, L. 2012, MNRAS, 427, 327

de la Torre, S., Guzzo, L., Kovač, K., et al. 2010, MNRAS, 409, 867

de la Torre, S., Guzzo, L., Peacock, J. A., et al. 2013, A\&A, 557, A54

Drinkwater, M. J., Jurek, R. J., Blake, C., et al. 2010, MNRAS, 401, 1429

Driver, S. P., Hill, D. T., Kelvin, L. S., et al. 2011, MNRAS, 413, 971

Dvali, G., Gabadadze, G., \& Porrati, M. 2000, Phys. Lett. B, 485, 208

Efstathiou, G., Sutherland, W. J., \& Maddox, S. J. 1990, Nature, 348, 705

Eisenstein, D. J., Zehavi, I., Hogg, D. W., et al. 2005, ApJ, 633, 560

Eisenstein, D. J., Weinberg, D. H., Agol, E., et al. 2011, AJ, 142, 72

Fritz, A., Scodeggio, M., Ilbert, O., et al. 2014, A\&A, 563, A92

Garilli, B., Le Fèvre, O., Guzzo, L., et al. 2008, A\&A, 486, 683

Garilli, B., Guzzo, L., Scodeggio, M., et al. 2014, A\&A, 562, A23

Granett, B. R., Guzzo, L., Coupon, J., et al. 2012, MNRAS, 421, 251

Guzzo, L., Pierleoni, M., Meneux, B., et al. 2008, Nature, 451, 541

Hammersley, P., Christensen, L., Dekker, H., et al. 2010, The Messenger, 142, 8

High, F. W., Stubbs, C. W., Rest, A., Stalder, B., \& Challis, P. 2009, AJ, 138, 110

Hinshaw, G., Larson, D., Komatsu, E., et al. 2013, ApJS, 208, 19

Ilbert, O., Arnouts, S., McCracken, H. J., et al. 2006, A\&A, 457, 841

Jain, B., \& Khoury, J. 2010, Ann. Phys., 325, 1479

Kaiser, N. 1987, MNRAS, 227, 1

Kauffmann, G., Heckman, T. M., White, S. D. M., et al. 2003, MNRAS, 341, 54
Komatsu, E., Dunkley, J., Nolta, M. R., et al. 2009, ApJS, 180, 330

Kwan, J., Lewis, G. F., \& Linder, E. V. 2012, ApJ, 748, 78

Lahav, O., Lilje, P. B., Primack, J. R., \& Rees, M. J. 1991, MNRAS, 251, 128

Laureijs, R., Amiaux, J., Arduini, S., et al. 2011 [arXiv: 1110.3193]

Le Fèvre, O., Saisse, M., Mancini, D., et al. 2003, in Proc. SPIE 4841, eds. M. Iye, \& A. F. M. Moorwood, 1670

Le Fèvre, O., Vettolani, G., Garilli, B., et al. 2005, A\&A, 439, 845

Lilly, S. J., Le Brun, V., Maier, C., et al. 2009, ApJS, 184, 218

Linder, E. V., \& Cahn, R. N. 2007, Astropart. Phys., 28, 481

Małek, K., Solarz, A., Pollo, A., et al. 2013, A\&A, 557, A16

Marchetti, A., Granett, B. R., Guzzo, L., et al. 2013, MNRAS, 428, 1424

Marulli, F., Bolzonella, M., Branchini, E., et al. 2013, A\&A, 557, A17

Monet, D. G., Levine, S. E., Canzian, B., et al. 2003, AJ, 125, 984

Peacock, J. A., \& Smith, R. E. 2000, MNRAS, 318, 1144

Peebles, P. J. E. 1980, The large-scale structure of the universe (Princeton University Press)

Percival, W. J., \& White, M. 2009, MNRAS, 393, 297

Percival, W. J., Baugh, C. M., Bland-Hawthorn, J., et al. 2001, MNRAS, 327, 1297

Percival, W. J., Reid, B. A., Eisenstein, D. J., et al. 2010, MNRAS, 401, 2148

Perlmutter, S., Aldering, G., Goldhaber, G., et al. 1999, ApJ, 517, 565

Pierre, M., Chiappetti, L., Pacaud, F., et al. 2007, MNRAS, 382, 279

Reid, B. A., \& White, M. 2011, MNRAS, 417, 1913

Reid, B. A., Samushia, L., White, M., et al. 2012, MNRAS, 426, 2719

Riess, A. G., Filippenko, A. V., Challis, P., et al. 1998, AJ, 116, 1009

Schlegel, D. J., Finkbeiner, D. P., \& Davis, M. 1998, ApJ, 500, 525

Scoccimarro, R. 2004, Phys. Rev. D, 70, 083007

Scodeggio, M., Franzetti, P., Garilli, B., et al. 2005, PASP, 117, 1284

Seljak, U. 2000, MNRAS, 318, 203

Skibba, R. A., Smith, M. S. M., Coil, A. L., et al. 2014, ApJ, 784, 128

Song, Y.-S., \& Percival, W. J. 2009, JCAP, 10, 4

Szalay, A. S., Connolly, A. J., \& Szokoly, G. P. 1999, AJ, 117, 68

Taruya, A., Nishimichi, T., \& Saito, S. 2010, Phys. Rev. D, 82, 063522

Weinberg, S. 1989, Rev. Mod. Phys., 61, 1

White, M., Song, Y., \& Percival, W. J. 2009, MNRAS, 397, 1348

Xia, J.-Q., Granett, B. R., Viel, M., et al. 2012, JCAP, 6, 10

York, D. G., Adelman, J., Anderson, Jr., J. E., et al. 2000, AJ, 120, 1579

Zehavi, I., Weinberg, D. H., Zheng, Z., et al. 2004, ApJ, 608, 16

Zhang, P., Liguori, M., Bean, R., \& Dodelson, S. 2007, Phys. Rev. Lett., 99, 141302

Zheng, Z., Coil, A. L., \& Zehavi, I. 2007, ApJ, 667, 760

1 INAF - Osservatorio Astronomico di Brera, via E. Bianchi 46, 23807 Merate/via Brera 28, 20122 Milano, Italy

e-mail: luigi.guzzo@brera.inaf.it

2 Dipartimento di Fisica, Università di Milano-Bicocca, Piazza della Scienza 3, 20126 Milano, Italy

3 INAF - Istituto di Astrofisica Spaziale e Fisica Cosmica (IASF) Milano, via Bassini 15, 20133 Milano, Italy

4 Aix - Marseille Université, CNRS, LAM (Laboratoire d'Astrophysique de Marseille) UMR 7326, 13388 Marseille, France

5 INAF - Osservatorio Astrofisico di Torino, 10025 Pino Torinese, Italy

6 Canada-France-Hawaii Telescope, 65-1238 Mamalahoa Highway, Kamuela HI 96743, USA

7 Aix-Marseille Université, CNRS, CPT (Centre de Physique Théorique) UMR 7332, 13288 Marseille, France

8 INAF - Osservatorio Astronomico di Bologna, via Ranzani 1, 40127, Bologna, Italy

9 Dipartimento di Matematica e Fisica, Università degli Studi Roma Tre, via della Vasca Navale 84, 00146 Roma, Italy

10 Institute of Cosmology and Gravitation, Dennis Sciama Building, University of Portsmouth, Burnaby Road, Portsmouth PO1 3FX, UK

11 Institute of Astronomy and Astrophysics, Academia Sinica, PO Box 23-141, 10617 Taipei, Taiwan

12 INAF - Osservatorio Astronomico di Trieste, via G. B. Tiepolo 11, 34143 Trieste, Italy

13 SUPA, Institute for Astronomy, University of Edinburgh, Royal Observatory, Blackford Hill, Edinburgh EH9 3HJ, UK

14 Institute of Physics, Jan Kochanowski University, ul. Swietokrzyska 15, 25-406 Kielce, Poland 
15 Department of Particle and Astrophysical Science, Nagoya University, Furo-cho, Chikusa-ku, 464-8602 Nagoya, Japan

16 Dipartimento di Fisica e Astronomia - Università di Bologna, viale Berti Pichat 6/2, 40127 Bologna, Italy

17 INFN, Sezione di Bologna, viale Berti Pichat 6/2, 40127 Bologna, Italy

18 Institut d'Astrophysique de Paris, UMR7095 CNRS, Université Pierre et Marie Curie, 98 bis boulevard Arago, 75014 Paris, France

19 Max-Planck-Institut für Extraterrestrische Physik, 84571 Garching b. München, Germany

20 Astronomical Observatory of the Jagiellonian University, Orla 171, 30-001 Cracow, Poland

21 National Centre for Nuclear Research, ul. Hoza 69, 00-681 Warszawa, Poland

22 Universitätssternwarte München, Ludwig-Maximillians Universität, Scheinerstr. 1, 81679 München, Germany
23 INAF - Istituto di Astrofisica Spaziale e Fisica Cosmica Bologna, via Gobetti 101, 40129 Bologna, Italy

24 INAF - Istituto di Radioastronomia, via Gobetti 101, 40129 Bologna, Italy

25 Università degli Studi di Milano, via G. Celoria 16, 20130 Milano, Italy

${ }^{26}$ INFN, Sezione di Roma Tre, via della Vasca Navale 84, 00146 Roma, Italy

27 INAF - Osservatorio Astronomico di Roma, via Frascati 33, 00040 Monte Porzio Catone, Italy

28 Laboratoire Lagrange, UMR 7293, Université de Nice SophiaAntipolis, CNRS, Observatoire de la Côte d'Azur, 06300 Nice, France

29 Astronomical Observatory of the University of Geneva, ch. d'Ecogia 16, 1290 Versoix, Switzerland 